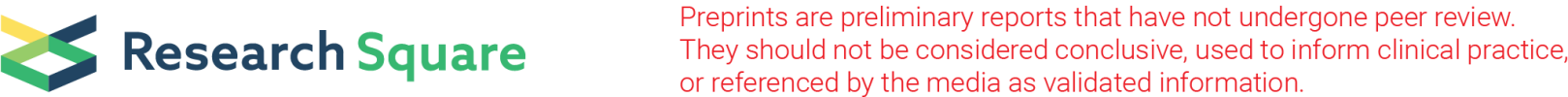

\section{Staphylococcal Superantigen-Like Protein 10 (SSL10) induces necroptosis through TNFR1 activation of RIPK3-dependent signal pathways}

Nan Jia

University of Science and Technology of China,

Guo Li

Fujian Medical University, Fuzhou

Wanbiao Chen

University of Science and Technology of China,

Chengliang Wang

University of Science and Technology of China,

Ling Chen

University of Science and Technology of China,

Xiaoling Ma

University of Science and Technology of China,

Xuan Zhang

University of Science and Technology of China,

Yue Tao

Jiao Tong University

Jianye Zang

University of Science and Technology of China,

Xi Mo ( $\nabla$ xi.mo@shsmu.edu.cn)

Jiao Tong University

Jinfeng Hu

Fujian Medical University

Article

Keywords: Staphylococcus aureus, SSL10, epithelial and endothelial systems, necroptosis, signaling pathways, crystal structure

Posted Date: May 5th, 2021

DOI: https://doi.org/10.21203/rs.3.rs-445262/v1 
License: (c) (i) This work is licensed under a Creative Commons Attribution 4.0 International License. Read Full License

Version of Record: A version of this preprint was published at Communications Biology on August 12th, 2022. See the published version at https://doi.org/10.1038/s42003-022-03752-8. 


\section{Abstract}

Staphylococcal aureus (S. aureus) infection can lead to a wide range of diseases such as sepsis and pneumonia. Staphylococcal superantigen like (SSL) proteins, specifically expressed by $S$. aureus, are shown to be involved in immune evasion during $S$. aureus infection. Here, we show that SSL10, an SSL family protein, exhibits potent cytotoxicity against human cells (HEK 293T and HUVEC) by inducing necroptosis upon binding to its receptor TNFR1 on the cell membrane. After binding, two distinct signaling pathways are activated downstream of TNFR1 in a RIPK3-dependent manner, i.e., the RIPK1RIPK3-MLKL and RIPK3-CaMKII-mitochondrial permeability transition pore (mPTP) pathways. We determined the crystal structure of SSL10 at $1.9 \AA$ resolution and, in conjunction with cytotoxicity analysis of chimeric mutant variants, identified a surface region formed by residues in the $\mathrm{N}$ - and Ctermini that can potentially serve as the TNFR1 binding site. Knockout of ss/10 in S. aureus profoundly reduced cytotoxicity of culture supernatants, indicating that SSL10 is involved in extracellular cytotoxicity during infection. This study thus provides the first description of cytotoxicity through induction of necroptosis by an SSL protein, and a potential target for clinical treatment of $S$. aureus-associated diseases.

\section{Introduction}

Staphylococcus aureus (S. aureus) is a prevalent and opportunistic pathogen that causes a wide range of diseases such as sepsis, pneumonia, endocarditis, and osteomyelitis, threatening the health of both humans and animals (1). Moreover, $S$. aureus is among the most clinically challenging pathogens worldwide because of its propensity for rapid development and sharing of antibiotic resistance (2). Although antibiotic treatments can reduce the case fatality rate of most $S$. aureus related diseases, some severe infectious diseases still have reported high mortality rates. For example, the case fatality rate for $S$. aureus bacteremia can range between 15 and 50\% (3). However, the mechanisms underlying these poor outcomes for some $S$. aureus-induced diseases have remained largely unknown.

S. aureus can manipulate host immune response through the expression of a myriad of virulence factors responsible for tissue adherence, immune evasion, cell injury, and organ failure, ultimately promoting its survival and pathogenesis $(2,4,5)$. The activities of some virulence factors have been shown to induce host cell death, especially through apoptosis and necroptosis, which facilitates immune evasion and tissue damage $(6,7)$. Necroptosis is a programmed form of necrosis that is regulated in a RIPK3 kinase signaling-dependent manner (8). Several types of receptors participate in the initiation stage of necroptosis, including death domain receptors, pathogen recognition receptors, and T cell receptors. Among these receptors, TNFR1 is well characterized for its role in triggering caspase-independent cell death via activation of RIPK1 and RIPK3 when stimulated by TNFa $(9,10)$. MLKL is an important downstream effector of RIPK3 due to its role in the formation of permeable cell membrane channels that lead to cell death (11). In addition, CaMKII is also phosphorylated by RIPK3, resulting in the opening of mitochondrial permeability transition pores (mPTPs) and subsequent necroptosis in cardiomyocytes, independent of MLKL (12). 
Activation of both necroptotic signaling pathways during $S$. aureus infection has been reported. For example, several of the $S$. aureus pore forming virulence factors can induce necroptosis in macrophages, which can lead to severe lung damage. Among them, a-hemolysin was demonstrated to instigate necroptosis mediated by MLKL (7). In addition, phagocytosis of $S$. aureus elicits necroptosis of neutrophils by activating RIPK3 in an MLKL- independent manner $(13,14)$. Moreover, in severe sepsis caused by infection of $S$. aureus infection, vascular permeation, immunosuppression, and organ failure are usually present, which strongly suggests the occurrence of cell death including necroptosis $(5,15,16)$.

Staphylococcal superantigen like (SSL) proteins comprise a family of 14 member proteins with sequences and structures homologous to superantigen but lacking superantigen activities. The genes encoding SSL proteins are located in a pathogenicity island in the genomes of all tested $S$. aureus strains (17-19). SSL proteins adopt conserved structures with an N-terminal OB-fold domain and a C-terminal $\beta$ grasp domain, which interact with diverse target factors to manipulate host immune response and interfere with blood coagulation in order to evade host defenses (20). For instance, SSL5 and SSL11 are able to bind sialylated sugar chains of P-selectin glycoprotein ligand 1 (PSGL-1) to inhibit neutrophil activation and rolling $(21,22)$. SSL7 interacts with IgA to block IgA-mediated immunity and binds complement component 5 (C5) to inhibit its activation $(23,24)$. SSL3 targets toll-like receptor 2 (TLR2) to prevent its activation by pathogen-associated molecular patterns (25). In contrast, SSL10 is a novel member of this family with distinct activities to bind CXCR4 and inhibit migration of leukemia cells (26), block the interactions between IgG and complement component $\mathrm{C} 1 \mathrm{q}$ that consequently prevent the activation of the classical complement pathway $(27,28)$, and interact with prothrombin and factor Xa to impair blood coagulation (29). Recent publications also show that SSL10 binds to ERK2, phosphatidylserine and apoptotic cells to either interfere host cell inflammation or procoagulant activity $(30,31)$. All these studies suggest that SSL10 possesses multiple functions during $S$. aureus infection, positing the importance of SSL10.

Although SSL proteins have diverse functions in modulating host response to $S$. aureus infection, it remains unknown whether SSL family proteins can induce cytotoxicity. In the present study, we demonstrate that SSL10 exhibits potent cytotoxicity towards HEK 293T and HUVEC cell growth by inducing cellular necroptosis and contributes to the cytotoxicity induced by $S$. aureus. We also propose an underlying mechanism for induction of necroptosis through binding with TNFR1, determined by crystal structure and modeling of protein binding interactions, and activating downstream RIPK3 signal pathways. This work provides evidence that SSL10 is a cytotoxic virulence factor that may serve as a therapeutic target in S. aureus infections.

\section{Results}

\section{SSL10 induces cell necrosis}

To determine the effects of SSLs on cells, human umbilical vein endothelial cells (HUVEC) were treated with purified recombinant SSLs protein, including SSL3, SSL7, SSL8, SSL10 or SSL11 for different time 
periods. As determined by MTS assay (Fig. S1), SSL10, but not other SSL family proteins we tested, significantly reduced the cell activity of HUVEC. Further, SSL10 treatment resulted in a decrease in cell viability in a dose- and time-dependent manner in human embryonic kidney cells (HEK 293T) and HUVEC, as determined by MTS assay (Fig. 1A and 1B). Most inviable cells were PI-positive, but were Annexin Vnegative when detected by flow cytometry, suggesting that cell death induced by SSL10 is most likely to be necrosis (Fig. 1C and 1D). Cell necrosis was further confirmed by transmission electron microscopy (TEM) in which the cells exhibited a typical necrotic phenotype, including cytoplasmic lightening, swollen organelle, and membrane rupture (Fig. 1E). SSL10 is a virulence factor secreted by S. aureus, so we quantified the cytotoxicity of supernatants from ss/10 knock out or complementation strain toward HEK 293T and HUVEC. We found that, compared with TSB medium-treated cells, LDH release was significantly greater in treatments with WT S. aureus 8325 supernatant. In contrast, supernatant from the ss/10 knock out strain, but not the ss/10 complemented strain, induced significantly decreased LDH release (Fig. 1F).

To further demonstrate that SSL10 induced necrosis rather than apoptosis, cells were treated by pancaspase inhibitor Z-VAD-fmk before exposure to SSL10, which resulted in no significant inhibition of cell death, as determined by $\mathrm{LDH}$ release (Fig. $1 \mathrm{G}$ and $1 \mathrm{H}$ ). As pyroptosis is also caspase dependent, our data thus suggest that SSL10 does not induce apoptosis or pyroptosis, but instead strongly implies activity by the primary cellular necrosis pathway.

\section{SSL10 induces necroptosis via the RIPK3-dependent pathway}

To explore the underlying mechanism by which SSL10 induced necrosis in HEK 293T and HUVEC cells, we next used different inhibitors to pretreat the cells prior to SSL10 exposure. As determined by LDH release, RIPK1 inhibitors (Nec-1 and Nec-1s) could only partially attenuate the effects of SSL10, while RIPK3 inhibitor (GSK'872) almost completely inhibited the necrotic effects of SSL10 (Fig. 2A and 2B). Thus, we speculated that SSL10 may induce cellular necroptosis. To further test this hypothesis, we generated knock out cell lines for key genes involved in the necroptosis pathway via CRISPR-Cas 9 in both HEK 293T and HUVEC cells (Fig. S2). Consistent with the effects of inhibitor treatment, knock out of RIPK3 but not RIPK1 or MLKL inhibited SSL10-induced necrosis (Fig. 2C and 2E) in HEK 293T cells. In addition, transient complementation with RIPK3 in RIPK3 ${ }^{-/-}$HEK 293T cells led to robust necrosis, evident by the release of LDH (Fig. 2F). Similarly, substantially less LDH was released in HUVECs knocked out for RIPK3 (Fig. 2D), indicating that SSL10 could induce RIPK3-dependent necroptosis in both HEK 293T and HUVECs.

\section{CaMKII activation and MPTP opening also contribute to SSL10-induced necroptosis}

Previous studies have reported that RIPK1 can form a complex with RIPK3, which further activates MLKL, resulting in necroptosis of several types of cells $(10,32)$. However, in the present study, we found that inhibition or knock out of RIPK1 or MLKL could not completely inhibit SSL10-induced necroptosis, suggesting that SSL10-induced necroptosis may also depend on other RIPK3-mediated pathways independent of RIPK1 and MLKL. 
In addition to MLKL, RIPK3 has been reported to phosphorylate CaMKII to induce the opening of mPTP channels, for example, leading to necroptosis in cardiomyocytes (12). To explore whether CaMKII is also involved in SSL10-induced necroptosis, HEK 293T and HUVEC cells were treated with KN-93, a selective inhibitor of CaMKII, prior to SSL10 treatment. As assessed by the release of LDH and ATP, inhibition of CaMKII profoundly abrogated SSL10-induced necroptosis (Fig. 3A-C); phospho-CaMKII levels were also significantly increased after SSL10 treatment (Fig. 3D), which suggested the involvement of CaMKII in SSL10-induced necroptosis. To further identify the downstream effector of CaMKII, we pretreated HEK 293T and HUVEC cells with an inhibitor of MPTP opening, CsA, which efficiently blocked SSL10-induced LDH release (Fig. 3E and 3F). In addition, SSL10 treatment led to mitochondrial depolarization, which was significantly hampered in the absence of RIPK3, evident by the decrease in mitochondrial membrane potential $\left(\Delta \Psi_{\mathrm{m}}\right)(\mathrm{Fig} .3 \mathrm{G})$, indicating that CaMKII-mPTP is also likely to be a primary candidate downstream pathway for RIPK3 in SSL10-induced necroptosis.

\section{SSL10 induces necroptosis by direct interaction with the TNFR1 extracellular domain (TNFR1 ${ }^{\text {ECD }}$ )}

Necroptosis is initiated through ligand binding to several receptors including TNFR1 (33-35). To explore whether SSL10 induces necroptosis by interacting with membrane receptors, SSL10 localization was observed by real-time live-cell analysis and scanning confocal microscopy. Notably, SSL10 was found to be enriched on the cell membrane within the first 30 min of treatment, suggesting that SSL10 may bind to a cell surface receptor (Fig. S3). TNFR1, as well as other TNF family death receptors, control necroptosis through activating RIPK1 and RIPK3 (36). To test whether TNFR1 was the receptor for SSL10, in vitro MBP pull-down assays were conducted using purified SSL10 and MBP-tagged TNFR1 ${ }^{\text {ECD }}$ (the extracellular domain of TNFR1 containing amino acids 22-211), which showed that SSL10 can indeedly interact with TNFR1 ${ }^{\mathrm{ECD}}$ (Fig. 4A).

To further confirm whether TNFR1 participates in SSL10-induced necroptosis, we knocked out TNFR1 in HEK 293T and HUVEC cells via CRISPR/Cas9, which blocked SSL10-induced necroptosis, as indicated by the significantly decreased release of LDH (Fig. 4B and 4C). We then confirmed that the essential role of TNFR1 in SSL10-induced cytotoxicity by flow cytometry of increased viable cell counts and decreased $\Delta \Psi_{\mathrm{m}}$ (Fig. 4D and 4E). Consistent with these findings, SSL10-binding to the HEK 293T cell surface was significantly reduced when TNFR1 was knocked out (Fig. 4F). Taken together, these data demonstrate that SSL10 activates cell necroptosis via direct interaction with the TNFR1 ECD.

\section{Overall structure of SSL10}

To further understand the molecular mechanisms driving the SSL10 activation of necroptosis through initiation of the TNFR1 signaling pathway, we next solved the crystal structure of SSL10 by molecular replacement at $1.9 \AA$ ̊ resolution. X-ray diffraction data and structure refinement statistics are shown in Table 1. SSL10 exists as a monomer in both solution and crystal lattice (Fig. S4). Two SSL10 molecules were observed in one asymmetric unit adopting approximately identical structures, with the RMSD value being $0.254 \AA$ when the two molecules are aligned (Fig. S5A). In light of these results, we select molecule $B$ for further investigation. SSL10 exhibits a typical superantigen-like structure, similar to other SSL 
family proteins, consisting of two distinct domains separated by a flexible linker region. The N-terminal OB-fold domain (residues 43-123) contains one a-helix, eight $\beta$-strands, and one $3_{10}$ helix, while the $C$ terminal $\beta$-grasp domain (residues 133-227) consists of one a-helix, seven $\beta$-strands, and two $3_{10}$ helices (Fig. 5A). Several, large, positively charged surface areas were identified in the SSL10 structure, whereas negatively charged regions were small (Fig. 5B). 
Table 1

Crystallographic data collection and structure refinement

SSL10

PDB code

\section{Data collection}

Space group

Cell dimensions

a, b, c $(\AA)$

$a, \beta, \gamma\left(^{\circ}\right)$

Resolution $(\AA)$

Wavelength $(\AA)$

Reflections (Unique)

Completeness (\%)

Overall $I / \sigma(I)$

Redundancy

$\mathrm{R}_{\text {sym }}$ or $\mathrm{R}_{\text {merge }}(\%)^{\mathrm{b}}$

\section{Refinement}

$\mathrm{R}_{\text {work }}{ }^{\mathrm{c}} / \mathrm{R}_{\text {free }}{ }^{\mathrm{d}}(\%)$

R.m.s deviations

Bond lengths $(\AA)$

Bond angles $\left(^{\circ}\right)$

Average B-factors $\left(\AA^{2}\right)$
6LWT

$P 2_{1} 2_{1} 2_{1}$

$41.24,71.21,140.66$

$90,90,90$

$50.0-1.90(1.93-1.90)^{a}$

0.9778

$214,863(33,517)$

$100.0(100.0)$

$16.2(7.0)$

6.4 (6.6)

$9.0(29.4)$

20.94 / 24.62

0.0088

1.3524

30.97

a The values in parentheses refer to statistics in the highest shell.

${ }^{\mathrm{b}} R_{\text {sym }}=\left|\mathrm{l}_{\mathrm{i}}<\right|>|/| \mathrm{l}_{\mathrm{i}} \mid$ where $\mathrm{I}_{\mathrm{i}}$ is the intensity of the ith measurement, and $\langle 1>$ is the mean intensity for that reflection.

${ }^{\mathrm{c}} R_{\text {work }}=\left|F_{P}-F_{P(\text { calc })}\right| / F_{P .}$.

${ }^{\mathrm{d}} R_{\text {free }}$ was calculated with $5.1 \%$ of the reflections in the test set.

e Statistics for the Ramachandran plot from an analysis using MolProbity. 


\section{SSL10}

\section{Ramachandran plot ${ }^{\mathrm{e}}$}

Most favored regions (\%)

96.23

Allowed regions (\%)

3.77

Generously allowed regions (\%)

0

a The values in parentheses refer to statistics in the highest shell.

${ }^{\mathrm{b}} R_{\text {sym }}=\left|\mathrm{I}_{\mathrm{i}}-<\right|>|/| \mathrm{I}_{\mathrm{i}} \mid$ where $\mathrm{I}_{\mathrm{i}}$ is the intensity of the ith measurement, and $<\mathrm{I}>$ is the mean intensity for that reflection.

${ }^{\mathrm{c}} R_{\text {work }}=\left|F_{P}-F_{P(\text { calc })}\right| / F_{P}$.

${ }^{\mathrm{d}} R_{\text {free }}$ was calculated with $5.1 \%$ of the reflections in the test set.

e Statistics for the Ramachandran plot from an analysis using MolProbity.

\section{Both the $\mathrm{N}$ - and C-terminal domains of SSL10 contribute to its cytotoxicity}

Among the SSL family proteins, SSL7 is the most similar member of SSL10, with the highest sequence identity to SSL10 and the RMSD value being $1.345 \AA$ when the structure of SSL10 was aligned to SSL7 (PDB code: 3KLS) (Fig. S5B).

To investigate which domain or domains of SSL10 may be critical for its cytotoxicity, we generated variants of SSL10 with the $\mathrm{N}$ - and C-terminal domains swapped between SSL7. We designated the two newly generated chimeric proteins as SSL7.10 and SSL10.7, with SSL7.10 containing the SSL7 Nterminus and the SSL10 C-terminus, and vice versa (Fig. 5C). We found that both of the two chimeric proteins could induce a marked release of LDH, which was less potent than SSL10, and SSL7 didn't induce any extra LDH release compared with the buffer-treated cells (Fig. 5D). In agreement with these results, MBP pull-down assays showed that both of the chimeric proteins, but not SSL7, could bind to the TNFR1 ${ }^{\mathrm{ECD}}$, and the binding of both proteins was weaker compared to that of SSL10 (Fig. 5E), indicating that both the $\mathrm{N}$ - and $\mathrm{C}$ - terminal domains participate in SSL10-induced necroptosis via interaction with TNFR1 ${ }^{\mathrm{ECD}}$.

\section{Potential binding site of SSL10 for TNFR1}

To understand the molecular mechanisms controlling SSL10 binding to TNFR1, the structure of TNFR1 ${ }^{\text {ECD }}$ (PDB code: 1EXT) was docked onto the structure of SSL10 using the HDOCK web server (http://hdock.phys.hust.edu.cn/) (Fig. 6A). In this model, eight residues including H64, K66, N85, S88, Q91, K206, K208, and Y209 of SSL10 suggested a potential binding region for TNFR1 ECD (Fig. 6A and 
6B). Among these residues, H64, K66, N85, S88, and Q91 are located in the N-terminal OB-fold domain, while the other three residues are found in the C-terminal $\beta$-grasp domain, consistent with the previous observation that both the $\mathrm{N}$ - and C-terminal domains of SSL10 contribute to its cytotoxicity (Fig. 5D). We then aligned the sequences of SSL10 and SSL7, which did not interact with TNFR1 in MBP pull-down assays, in order to investigate differences in the eight residues between the two proteins (Fig. 6C). In SSL7, the corresponding residues are N68, S70, K89, D92, K95, Q208, E210, and R211, respectively, which differed from the charge of those amino acids in SSL10.

To further verify the binding model of SSL10 to the TNFR1 1 ECD , we generated two mutant variants, by replacing the eight residues $\mathrm{H64}, \mathrm{K} 66, \mathrm{~N} 85, \mathrm{~S} 88, \mathrm{Q} 91, \mathrm{~K} 206, \mathrm{~K} 208$, and Y209 in SSL10 with either alanine residues (mutant $\mathrm{A}$ ) or with the corresponding residues from SSL7 (mutant $\mathrm{B}$ ). The results of LDH assays showed that both mutants exhibited a significant reduction in LDH release compared with that in SSL10, and with mutant B having the weakest cytotoxicity (Fig. 6D). Supporting these results, binding by either mutant to TNFR1 ${ }^{\mathrm{ECD}}$ was weaker than that of SSL10 in MBP pull-down assays, nearly $67 \%$ and $49 \%$ compared to SSL10, respectively (Fig. 6E and 6F). Therefore, the docking and mutagenesis analyses demonstrate that residues $\mathrm{H64}, \mathrm{K} 66, \mathrm{~N} 85, \mathrm{~S} 88, \mathrm{Q} 91, \mathrm{~K} 206, \mathrm{~K} 208$, and $\mathrm{Y} 209$ are critical for SSL10 binding with TNFR1 ${ }^{\mathrm{ECD}}$ and subsequent initiation of the necroptosis signal cascade.

\section{Discussion}

Previous studies on SSL10, one of the SSL proteins specifically expressed in S. aureus, suggested that it contributed to $S$. aureus infection though inhibiting the classical complement activation pathway, the migration of $\mathrm{T}$ cells, the interaction between complement $\mathrm{C} 1 \mathrm{q}$ and $\mathrm{IgG}$, and the Fc-receptor-mediated phagocytosis of neutrophils (26-29). In addition, recent publications demonstrated that SSL10 binds to ERK2, phosphatidylserine and apoptotic cells to either interfere host cell inflammation or procoagulant activity $(30,31)$. Here, our data demonstrated the cytotoxicity of SSL10 by triggering necroptosis via activation of two distinct signaling pathways by binding to TNFR1 in HET 293T and HUVEC cells.

Though RIPK3 is expressed at an extremely low level in endothelial cell, including HUVEC, and HEK 293T, its importance in vivo cannot be excluded $(37,38)$. Genetic evidence showed that RIPK3 deficiency leads to reduced endothelial cell permeability or necroptosis, thereby suppressing tumor metastasis $(39,40)$. Furthermore, RIPK3 can be induced or upregulated under certain conditions, which confers cells sensitive to RIPK3-dependent necroptosis (39). Notably, we found an SSL10-induced increase in the protein level of RIPK3 in HEK 293T and HUVEC cells (Fig. S6), indicating the important role of RIPK3 on SSL10-induecd necroptosis in HEK 293T and HUVEC.

The best-characterized mechanism of necroptosis is RIP1-RIP3-MLKL signal pathway, which is induced by the interaction of TNF- $a$ and its receptor TNFR1 (32). More recently, CaMKII has been identified as a direct substrate of RIP3 to appropriate downstream effector MPTP in myocardial necroptosis (12). Opening of the MPTP results in loss of mitochondrial inner membrane potential, disruption of ATP production, increased ROS production, organelle swelling, mitochondrial dysfunction and consequent 
necrosis (41). Notably, the upstream receptor involved in RIPK3-CaMKII pathway is still unknown. Here, we found that TNFR1 contributed to RIPK3-CaMKII pathway induced by SSL10, but the mechanism underlying how TNFR1 activates RIPK3-CaMKII needs further study.

X-ray crystallography revealed SSL10 possess the characteristic structure of SSL family proteins, which consist of an N-terminal OB-fold and C-terminal $\beta$-grasp domain $(42,43)$. The OB-fold domain is responsible for recognition of protein ligands, whereas the $\beta$-grasp domain is capable of binding to tetrasaccharide sialyl Lewis $X(22,23,25,42-46)$. For example, SSL7 binds to the human IgA1 Fc domain through the N-terminal a helix and the L1 and L4 loops of the OB-fold domain, which results in steric shielding of the FcaRI binding site and inhibits FcaRI-mediated immunity (23). In addition, SSL3 interacts with TLR2 (to prevent its lipopeptide binding and dimerization) via four loops localized in the OB-fold domain, leading to blockade of TLR2 signaling and immune evasion (25). However, different from those previously reported SSL proteins, both the L1 loop and $3_{10} 1$ helix in the OB-fold domain, as well as $3_{10} 2$ helix in $\beta$-grasp domain of SSL10 participate in forming a surface for TNFR1 binding, which contributes to the activation of TNFR1 signaling-mediated necroptosis (Figs. 5 and 6 ). A recent report showed that two sequences from both the $\mathrm{N}$ - and C-terminal domains of SSL10 were involved in binding to prothrombin (47). Mapping onto the structure of SSL10 showed that the L1 loop and $3_{10} 2$ helix are localized in these two regions. According to these observations, SSL10 likely employs a different mechanism for binding partner recognition than that of other SSL proteins. Notably, further studies, informed by the work reported here, are needed to solve the complex structure of SSL10-TNFR1 ECD, so that we can identify the exact binding sites and key residues.

Damage of endothelial cells can facilitate the spread of $S$. aureus into the bloodstream and then may cause the development of septic shock and organ failure $(48,49)$. Virulence factors from $S$. aureus play important roles in the development and progression of sepsis through multiple mechanisms including disrupting various types of host cells $(4,6,50-53)$. Reijer et al. characterized the serial levels of IgG and IgA antibodies against 56 staphylococcal antigens in multiple serum samples of 21 patients with a $S$. aureus bacteremia. Their data showed that an increase in IgG levels against SSL10 was observed at some time point after the onset of bacteremia in 95 to $100 \%$ of all patients (54). Haemostatic abnormalities frequently occur during sepsis and are most often attributed to disseminated intravascular coagulation (DIC). Draaijers et al. reported the case of a patient with severe coagulopathy acquired during fulminant $S$. aureus sepsis and speculated that inhibition of coagulation factor $X$ by $S$. aureus SSL 10 is the most likely cause of the acquired coagulopathy in their patient (55). Above, in combination with our data, we suggest that SSL10 may be involved in the progress of $S$. aureus sepsis by different mechanisms.

In summary, we provide the first demonstration of which we are aware that SSL10 can act as a signal to initiate necroptotic programmed cell death signal via direct interaction with TNFR1 in HEK 293T and HUVEC. Moreover, this signal cascade is activated in a RIPK3-dependent manner but is transduced through two independent signaling pathways (Fig. 7). Using molecular docking with the structure of TNFR1 ${ }^{E C D}$ to SSL10, we identified a surface region of SSL10 as the potential binding site for TNFR1. 
Thus, this study, in combination with other previous reports, provides strong evidence that SSL10 contributes to $S$. aureus infection via multiple mechanisms, and suggest this virulence factor may serve as a potentially reliable biomarker and therapeutic target for $S$. aureus-associated infection and diseases.

\section{Materials And Methods}

\section{Reagents and cell culture}

Human umbilical vein endothelial cell line (HUVEC) and human embryonic kidney cell line (HEK 293T) were obtained from Cell Bank of Chinese Academy of Sciences (Shanghai, China) and were cultured according to its instructions. The cells were cultured in Dulbecco's Modified Eagle's Medium (DMEM) supplemented with $10 \%$ fetal bovine serum (FBS) and incubated at $37^{\circ} \mathrm{C}$ in humidified incubator with $5 \%$ $\mathrm{CO}_{2}$. S. aureus RN4220 and S. aureus 8325 strains were kindly provided by Dr. Min Li (Renji Hospital, Shanghai Jiao Tong University School of Medicine).

Primary antibodies against RIPK1, RIPK3, MLKL, TNFR1, CaMKII, phosph-CaMKII and GAPDH were purchased from Cell Signaling Technology (Danvers, MA, USA). Primary antibodies against actin and tubulin were purchased from TransGen Biotechnology (Beijing, China). The secondary antibodies including anti-rabbit IgG H\&L (HRP-conjugated) and anti-mouse IgG H\&L (HRP-conjugated) were obtained from Beyotime Biotechnology (Shanghai, China).

CellTiter 96® AQueous One Solution Cell Proliferation Assay and CytoTox 96® Non-Radioactive Cytotoxicity Assay (Promega, Madison, WI) were used for MTS and Lactate dehydrogenase (LDH) assays, respectively. To measure the ATP concentration in the cells, CellTiter-Glo ${ }^{\circledR}$ Luminescent Viability Assay (Promega, Madison, WI) was used, while FITC Annexin V Appoptosis Detection KIT I (BD, New Jersey, US) was used to determine the cell death by flow cytometry.

Inhibitors used in the present study are as follows: necrostatin-1 (Nec-1, Roche), necrostatin-1 stable (Nec1s, Biovision), GSK'872 (Calbiochem), Z-VAD-fmk (Selleckchem), KN-93 (APExBIO), and cyclosporine A (CsA, APExBIO).

\section{Cytotoxicity Assays}

HUVECs or HEK 293T cells were seeded in $96-$ well plate $\left(100 \mu \mathrm{L}\right.$ per well) at a density of $1 \times 10^{4}$ cells/well one day prior to treatment with or without SSL10 resuspended in Opti-MEM reduced serum medium (Thermo Fisher) for $48 \mathrm{~h}$. To detect the effects of inhibitors, the cells were pretreated with various inhibitors $0.5 \mathrm{~h}$ before $2 \mu \mathrm{M}$ SSL10 treatment.

For MTS assay, $20 \mu \mathrm{L}$ of CellTiter $96 \AA$ AQueous One Solution reagent was added to each well and incubated for two more hours, and the optical density was measured at $490 \mathrm{~nm}$ with a BioTek Synergy/2 microplate reader (BioTek, Winooski, VT). 
LDH release was measured according to the manufacturer's manual. Briefly, $50 \mu \mathrm{L}$ culture media from various treated cells were transferred to a new 96 -well flat clear bottom plate, and $50 \mu \mathrm{L}$ of the CytoTox $96 \AA$ reagent was added to each sample aliquot and incubated in dark for $30 \mathrm{~min}$ at room temperature. Finally, $50 \mu \mathrm{L}$ of stop solution was added to each well and the absorbance at $490 \mathrm{~nm}$ was recorded with BioTek Synergy/2. The level of LDH released was expressed as a fold of the control (buffer-treated cells) group after subtracting the background absorbance.

To determine the ATP concentration in the cells, CellTiter-Glo® reagent was added to each well and the plate was incubated for $10 \mathrm{~min}$ to stabilize luminescent signal before luminescence being recorded with BioTek Synergy/2. Luminescent signals from blank wells and buffer-treated cells were used as background and maximal luminescence.

Cell death detected by flow cytometry was performed as previously described (56). Briefly, $48 \mathrm{~h}$ after treatment with SSL10, the cells were collected and washed twice with ice-cold PBS. The cells were then incubated with $5 \mu \mathrm{L}$ FITC annexin V and $5 \mu \mathrm{L} \mathrm{PI} \mathrm{in} 500 \mu \mathrm{L}$ prepared assay buffer in dark for $10 \mathrm{~min}$ at room temperature, and applied for flow cytometry analysis.

\section{Knockout and rescue of ss/10 in S. aureus 8325}

To explore the effect of SSL10 on the cytotoxicity of S. aureus, ss/10-knockout strain was constructed using the vector pKOR1 as previously described (57). Briefly, 1, 000 bp fragment upstream and downstream, respectively, of ss/10 was cloned to PKOR1 via lambda recombination (BP clonase enzyme mix, Invitrogen). The resulting plasmid was transferred via electroporation first to $S$. aureus RN4220 to modify DNA, and subsequently to $S$. aureus 8325 . For homologous recombination and ingratiation of pKOR1 into the bacterial chromosome, $S$. aureus 8325 was grown at $43^{\circ} \mathrm{C}$ on tryptic soy agar $\left(\mathrm{TSA}_{\mathrm{Cm} 10}\right)$, a non-permissive condition for pKOR1 replication. From the resulting plate, one colony was picked, inoculated into $1 \mathrm{ml} \mathrm{TSB} \mathrm{Cm} 10_{10}$ and incubated at $30^{\circ} \mathrm{C}$ overnight to facilitate plasmid excision. Cultures were then spread on TSA containing $200 \mathrm{ng} / \mathrm{mL}$ anhydrotetracycline and incubated at $30^{\circ} \mathrm{C}$ overnight for selecting ss/10-knockout $S$. aureus 8325 . To rescue $s s / 10$ in ss/10 knockout strain, the gene sequence of $s s / 10$ was ligated with $h p r k$ promoter and then cloned to the plasmid pOS1. The resulting plasmid was transferred via electroporation first to $S$. aureus RN4220 to modify DNA, and subsequently to ss/10knockout S. aureus 8325.

\section{Cytotoxicity Assay of S. aureus ss/10-knockout or Rescue Strains}

S. aureus 8325 wild type, ss/10-knockout and rescue strains were cultured in TSB medium (OXOID) for $8 \mathrm{~h}$ at $37^{\circ} \mathrm{C}, 220 \mathrm{rpm}$. The same number of bacteria cells were inoculated into a new tube of TSB medium respectively, and cultured overnight at $37^{\circ} \mathrm{C}, 220 \mathrm{rpm}$ for protein expression and secretion. Bacteria cells were collected after achieving stagnate phase and the supernatants were obtained by centrifugation at 5 , $000 \mathrm{rpm}$ for $15 \mathrm{~min}$ at room temperature. After filtration with a $0.22 \mu \mathrm{m}$ filter, the supernatants were used to treat HEK 293T or HUVEC cells for $48 \mathrm{~h}$ after 1:1, 000 dilution with Opti-MEM reduced serum medium, 
and $L D H$ released from the cells were determined. The level of LDH released was expressed as the fold of the control group (TSB medium-treated cells) after subtracting the background absorbance.

\section{Transmission Electron Microscopy}

HEK 293T or HUVEC cells with or without SSL10 treatment were prefixed in Karnovsky's solution (1\% paraformaldehyde, $2 \%$ glutaraldehyde, $2 \mathrm{mM}$ calcium chloride, $0.1 \mathrm{M}$ cacodylate buffer, $\mathrm{pH} 7.4$ ) for $2 \mathrm{~h}$ and washed with cacodylate buffer. Postfixing was carried out in $1 \%$ osmium tetroxide and $1.5 \%$ potassium ferrocyanide for $1 \mathrm{~h}$. After dehydration with $50-100 \%$ alcohol, the cells were embedded in Poly/Bed 812 resin (Pelco, Redding, CA, USA), polymerized and then observed under a HITACHI model H7650 electron microscope (HITACHI, Tokyo, Japan).

\section{Western Blotting}

The cells were lysed in RIPA buffer containing proteinase inhibitor (Sigma-Aldrich, St. Louis, MO, USA) and/or PhosSTOP Phosphatase Inhibitor (Roche, Basel, Switzerland) when necessary. A total of $30 \mu \mathrm{g}$ of protein (determined by BCA protein quantification kit) for each sample was separated on $10 \%$ SDS-PAGE, transferred to a PVDF membrane, immunoblotted with appropriate antibodies. Antibody binding was detected using a luminescent image analyzer ImageQuant ${ }^{\text {TM }}$ LAS 4000 mini (GE Healthcare Bio-Science $A B$, Uppsala, Sweden) after adding peroxidase-conjugated secondary antibodies and chemiluminescence substrates.

\section{CRISPR-Cas9 Genome Editing and Rescue}

The E-CRISP online tool (http://www.e-crisp.org/E-CRISP/) was used to design specific single-guide RNAs targeting different genes. The 20-nucleotide guide sequence was annealed to the complementary oligos and then cloned into the pSpCas9(BB)-2A-green fluorescent protein (GFP) plasmid (PX458; Addgene, Cambridge, MA, USA). HEK 293T or HUVEC cells were transiently transfected with the CRISPR/Cas9 plasmids using Lipofectamine 3000 (Life Technologies, MA, USA) following the manufacturer's instructions. GFP-positive single clone was sorted using SmartSampler Analyzer (Beckman, California, USA) $48 \mathrm{~h}$ after transfection and cultured in a 96-well plate for about 10 days before confirming the gene knockout by sequencing and western blotting or flow cytometry. Sequences of the sgRNAs for various genes are listed in the Supplementary Materials Table S1.

For RIPK3 rescue, RIPK3 cDNA was cloned into the pCDH-CMV-MCS-EF1-copGFP-T2A-Puro vector (Addgene, Cambridge, MA, USA). HEK 293T WT and RIPK3-KO cells were transiently transfected with the RIPK3-expression plasmids using Lipofectamine 3000, and $48 \mathrm{~h}$ after transfection, SSL10-induced necroptosis was detected by LDH release assay.

\section{Mitochondrial Membrane Potential Assay}

The mitochondrial potential, which reflects mitochondrial depolarization, was detected using the mitochondrial membrane potential assay kit following the manufacturer's instructions (Beyotime, Shanghai, China). Briefly, JC-1 working solution was incubated with the cells in dark for 20 min at $37^{\circ} \mathrm{C}$. After three washes with prepared buffer, the cells were detected on a flow cytometer using PE and FITC 
channels. The value of the $\mathrm{JC}-1$ monomers to aggregates positive cells ratio quantifies the mitochondrial membrane depolarization.

\section{Real-time Live-cell Analysis}

HUVECs were seeded in 96-well plate $\left(100 \mu \mathrm{L}\right.$ per well) at a density of $1 \times 10^{3}$ cells/well with or without 2 $\mu \mathrm{M}$ GFP-SSL10/GFP treatment and then observed with live-cell dynamic imaging and analysis system (Incucyte S3, Essen Bioscience, USA).

\section{Laser Scanning Confocal Microscope}

HUVECs were seeded on coverslips in a 24-well plate and then incubated for $4 \mathrm{~h}$ before being incubated with $2 \mu \mathrm{M}$ GFP-SSL10 protein resuspended in Opti-MEM medium for $20 \mathrm{~min}$. After treatment, the cells were washed twice with PBS to remove unbound proteins and then fixed with $4 \%$ paraformaldehyde for 20 min. After fixation, cells were washed three times with PBS and then incubated with DAPI. After three additional PBS washes, coverslips were mounted onto slides using antifade mounting medium (Beyotime, Shanghai, China). The cells were visualized using a laser scanning confocal microscope (Leica, Wetzlar, Germany).

\section{Protein Expression and Purification}

DNA fragments encoding amino acid residues $31-227$ of SSL10 or its mutants were amplified by PCR from S. aureus strain Mu50 and cloned into the pET-22b (+) vector (Novagen) with a C-terminal $6 \times$ His tag. Wild type and mutant SSL10 were expressed in Escherichia coli BL21 (DE3) and induced with $0.4 \mathrm{mM}$ IPTG (isopropyl $\beta$-D-1-thiogalactopyranoside) for $4 \mathrm{~h}$ at $37^{\circ} \mathrm{C}$ when $\mathrm{OD}_{600}$ reached 0.6 . The cells were harvested by centrifugation at 6,000 rpm for $8 \mathrm{~min}$, and lysed in a French press in lysis buffer [50 mM Tris- $\mathrm{HCl}, \mathrm{pH} 7.5,500 \mathrm{mM} \mathrm{NaCl}, 5 \%$ (v/v) Glycerol, $5 \mathrm{mM}$ imidazole, $1 \mathrm{mM}$ PMSF]. The lysate was centrifuged at 15, $000 \mathrm{rpm}$ for $30 \mathrm{~min}$, and the supernatant was incubated with Ni-NTA resin for $30 \mathrm{~min}$. The resin with target proteins were washed with 50 column volumes of washing buffer [ $50 \mathrm{mM}$ Tris-HCl, $\mathrm{pH} 7.5,500 \mathrm{mM} \mathrm{NaCl}, 5 \%(\mathrm{v} / \mathrm{v})$ Glycerol, $40 \mathrm{mM}$ imidazole] to remove contaminants and the target protein was eluted by elution buffer [50 mM Tris- $\mathrm{HCl}, \mathrm{pH} 7.5,500 \mathrm{mM} \mathrm{NaCl}, 5 \%$ (v/v) Glycerol, $300 \mathrm{mM}$ imidazole]. The eluted protein was concentrated and further purified by Superdex 75 10/300 size exclusion column (GE Healthcare) equilibrated with the buffer containing $20 \mathrm{mM}$ Tris-HCl, pH 7.5 and $200 \mathrm{mM} \mathrm{NaCl}$. The entire protein purification procedure was carried out at $4^{\circ} \mathrm{C}$. Purity of the target protein was verified by SDS-PAGE and protein aliquots were stored at $-80^{\circ} \mathrm{C}$ for further use.

GFP-SSL10 used for laser scanning confocal microscopy was cloned into the pET-28a(+) vector (Novagen) with the GFP- and 6xHis-tag fused to its N-terminus. The protein was expressed and purified using the method similar as above.

DNA fragment of human TNFR1 extracellular domain (amino acid residues 22-211) was amplified by $\mathrm{PCR}$ and cloned into the $\mathrm{pET}-28 \mathrm{a}(+)$ vector with an N-terminal MBP-tag. The recombinant protein MBPTNFR1 ${ }^{\mathrm{ECD}}$ was expressed in E. coli Rosetta2 (DE3) strain and purified by MBP-affinity chromatography. 
SSL10 was concentrated to $4.3 \mathrm{mg} / \mathrm{mL}$ (in buffer containing $20 \mathrm{mM}$ Tris- $\mathrm{HCl}, \mathrm{pH} 7.5$ and $200 \mathrm{mM} \mathrm{NaCl}$ ), and used for initial crystallization trials by the sitting-drop vapor-diffusion method at $16^{\circ} \mathrm{C}$ with index crystallization screen kit. Crystals were obtained from the buffer containing 2.1 M DL-malic acid, pH 7.0.

\section{Data Collection, Structure Determination and Refinement}

The crystals of SSL10 were soaked in cryoprotectant buffer consisting 2.1 M DL-Malic acid, pH 7.0 and $20 \%$ Glycerol for several seconds and flash-cooled in liquid nitrogen. X-ray diffraction data was collected at beamline BL18U1 of Shanghai Synchrotron Radiation Facility (SSRF). Diffraction data were processed, integrated, and scaled using HKL2000 (58).

The crystal structure of SSL10 was determined by molecular replacement using the program Phaser in the CCP4i suite $(59,60)$ with Exotoxin SACOL0473 (PDB code 3R2I) as the search model. After several runs of structure refinement using the programs REFMAC5, Phenix and Coot (61-63), the final model was refined to $1.9 \AA$ resolution with $R_{\text {work }}$ of $20.94 \%$ and $R_{\text {free }}$ of $24.62 \%$. Data collection and structure refinement statistics are summarized in Table 1. All figures of protein structure were prepared using PyMOL (http://www.pymol.org).

\section{MBP Pull-down Assay}

Thirty microgram of MBP-TNFR1 ${ }^{E C D}$ was incubated with $30 \mu \mathrm{g}$ of wild type or mutant SSL10 for $1 \mathrm{~h}$ on ice, and the protein mixture was centrifuged at $15,000 \mathrm{rpm}$ for $30 \mathrm{~min}$ at $4^{\circ} \mathrm{C}$ to remove precipitates. The supernatant was then added into $1 \mathrm{~mL}$ binding buffer $(20 \mathrm{mM}$ Tris- $\mathrm{HCl}, \mathrm{pH} 7.5,200 \mathrm{mM} \mathrm{NaCl}, 0.5 \%$ NP40) with $20 \mu \mathrm{L}$ MBP beads, and incubated for $1 \mathrm{~h}$ at $4^{\circ} \mathrm{C}$. The beads were washed with $1 \mathrm{~mL}$ binding buffer for four times to remove any non-specific bindings, and the proteins bound to MBP resin were eluted by $20 \mu \mathrm{L}$ elution buffer ( $20 \mathrm{mM}$ Tris-HCl, pH 7.5, $200 \mathrm{mM} \mathrm{NaCl}, 50 \mathrm{mM}$ D-Maltose) and analyzed by $15 \%$ SDS-PAGE.

\section{Statistical Analysis}

All data were collected from at least three independent experiments, and presented as the mean \pm SD. Comparisons between two groups were analyzed by the multiple $t$ test or by two-way ANOVA using GraphPad Prism Version 6.0 (GraphPad Inc., La Jolla, CA, USA) software program. $p$ values $<0.05$ were considered statistically significant.

\section{Declarations}

\section{Acknowledgements}

The authors would like to thank the staff at beamline BL18U1 of the Shanghai Synchrotron Radiation Facility of the National Facility for Protein Science in Shanghai for the assistance with data collection. This work was supported by the National Natural Science Foundation of China (81801974 to JH, 91853133 to JZ, 31700671 to XZ, 81701936 to YT, and 81971890 to XM), the Natural Science Foundation of Fujian Province of China (2020J01615), the National Key Research and Development Program of China (2017YFA0503600, 2016YFA0400903), the Foundation for Innovative Research Groups 
of the National Natural Science Foundation of China (31621002), Shanghai Key Laboratory of Clinical Molecular Diagnostics for Pediatrics (20dz2260900), Shanghai Key Laboratory of Emergency Prevention, Diagnosis and Treatment of Respiratory Infectious Diseases (20dz2261100), the Innovative Program of Development Foundation of Hefei Center for Physical Science and Technology (2017FXCX004), Users with Excellence Project of Hefei Science Center CAS (2018HSC-UE001), China Postdoctoral Science Foundation (2017M621492), FJMU Scientific Research Foundation (XRCZX2019013), and USTC Research Funds of the Double First-Class Initiative.

Competing interests: The authors declare no competing interests.

\section{References}

1. RJ M. Recognition and management of Staphylococcus aureus toxin-mediated disease. Intern Med J. 2005;35:S106-S19.

2. Turner NA, Sharma-Kuinkel BK, Maskarinec SA, Eichenberger EM, Shah PP, Carugati M, et al. Methicillin-resistant Staphylococcus aureus: an overview of basic and clinical research. Nat Rev Microbiol. 2019;17(4):203-18.

3. Tong SY, Davis JS, Eichenberger E, Holland TL, Fowler VG, Jr. Staphylococcus aureus infections: epidemiology, pathophysiology, clinical manifestations, and management. Clin Microbiol Rev. 2015;28(3):603-61.

4. Thammavongsa V, Kim HK, Missiakas D, Schneewind O. Staphylococcal manipulation of host immune responses. Nat Rev Microbiol. 2015;13(9):529-43.

5. Lelubre C, Vincent JL. Mechanisms and treatment of organ failure in sepsis. Nat Rev Nephrol. 2018;14(7):417-27.

6. Yang L, Zhou X, Huang W, Fang Q, Hu J, Yu L, et al. Protective Effect of Phillyrin on Lethal LPSInduced Neutrophil Inflammation in Zebrafish. Cell Physiol Biochem. 2017;43(5):2074-87.

7. Kitur K, Parker D, Nieto P, Ahn DS, Cohen TS, Chung S, et al. Toxin-induced necroptosis is a major mechanism of Staphylococcus aureus lung damage. PLoS Pathog. 2015;11(4):e1004820.

8. Galluzzi L, Kepp O, Chan FK, Kroemer G. Necroptosis: Mechanisms and Relevance to Disease. Annu Rev Pathol. 2017;12:103-30.

9. Holler N, Zaru R, Micheau O, Thome M, Attinger A, Valitutti S, et al. Fas triggers an alternative, caspase-8-independent cell death pathway using the kinase RIP as effector molecule. Nat Immunol. 2000;1(6):489-95.

10. Cho YS, Challa S, Moquin D, Genga R, Ray TD, Guildford M, et al. Phosphorylation-driven assembly of the RIP1-RIP3 complex regulates programmed necrosis and virus-induced inflammation. Cell. 2009;137(6):1112-23.

11. Cai Z, Jitkaew S, Zhao J, Chiang HC, Choksi S, Liu J, et al. Plasma membrane translocation of trimerized MLKL protein is required for TNF-induced necroptosis. Nat Cell Biol. 2014;16(1):55-65. 
12. Zhang T, Zhang $Y$, Cui $M$, Jin L, Wang Y, Lv F, et al. CaMKII is a RIP3 substrate mediating ischemiaand oxidative stress-induced myocardial necroptosis. Nat Med. 2016;22(2):175-82.

13. Greenlee-Wacker MC, Rigby KM, Kobayashi SD, Porter AR, DeLeo FR, Nauseef WM. Phagocytosis of Staphylococcus aureus by human neutrophils prevents macrophage efferocytosis and induces programmed necrosis. J Immunol. 2014;192(10):4709-17.

14. Greenlee-Wacker MC, Kremserova S, Nauseef WM. Lysis of human neutrophils by communityassociated methicillin-resistant Staphylococcus aureus. Blood. 2017;129(24):3237-44.

15. Choi ME, Price DR, Ryter SW, Choi AMK. Necroptosis: a crucial pathogenic mediator of human disease. JCI Insight. 2019;4(15):e128834.

16. Powers ME, Bubeck Wardenburg J. Igniting the fire: Staphylococcus aureus virulence factors in the pathogenesis of sepsis. PLoS Pathog. 2014;10(2):e1003871.

17. Kuroda M, Ohta T, Uchiyama I, Baba T, Yuzawa H, Kobayashi I, et al. Whole genome sequencing of meticillin-resistant Staphylococcus aureus. Lancet. 2001;357(9264):1225-40.

18. Fitzgerald JR, Reid SD, Ruotsalainen E, Tripp TJ, Liu M, Cole R, et al. Genome diversification in Staphylococcus aureus: Molecular evolution of a highly variable chromosomal region encoding the Staphylococcal exotoxin-like family of proteins. Infect Immun. 2003;71(5):2827-38.

19. Aguiar-Alves F, Medeiros F, Fernandes O, Gudziki Pereira RM, Perdreau-Remington F, Riley LW. New Staphylococcus aureus genotyping method based on exotoxin (set) genes. J Clin Microbiol. 2006;44(8):2728-32.

20. Fraser JD, Proft T. The bacterial superantigen and superantigen-like proteins. Immunol Rev. 2008;225:226-43.

21. Bestebroer J PM, Ulfman LH, Lenting PJ, Denis CV, van Kessel KP, van Strijp JA, de Haas CJ. Staphylococcal superantigen-like 5 binds PSGL-1 and inhibits P-selectin-mediated neutrophil rolling. Blood. 2007.

22. Chung MC, Wines BD, Baker H, Langley RJ, Baker EN, Fraser JD. The crystal structure of staphylococcal superantigen-like protein 11 in complex with sialyl Lewis $\mathrm{X}$ reveals the mechanism for cell binding and immune inhibition. Mol Microbiol. 2007;66(6):1342-55.

23. Ramsland PA, Willoughby N, Trist HM, Farrugia W, Hogarth PM, Fraser JD, et al. Structural basis for evasion of IgA immunity by Staphylococcus aureus revealed in the complex of SSL7 with Fc of human IgA1. Proc Natl Acad Sci U S A. 2007;104(38):15051-6.

24. Laursen NS, Gordon N, Hermans S, Lorenz N, Jackson N, Wines B, et al. Structural basis for inhibition of complement C 5 by the SSL7 protein from Staphylococcus aureus. Proc Natl Acad Sci U S A. 2010;107(8):3681-6.

25. Koymans KJ, Feitsma LJ, Brondijk THC, Aerts PC, Lukkien E, Lössl P, et al. Structural basis for inhibition of TLR2 by staphylococcal superantigen-like protein 3 (SSL3). Proceedings of the National Academy of Sciences. 2015;112(35):11018-23.

26. Walenkamp AM, Boer IG, Bestebroer J, Rozeveld D, Timmer-Bosscha H, Hemrika W, et al. Staphylococcal superantigen-like 10 inhibits CXCL12-induced human tumor cell migration. 
Neoplasia. 2009;11(4):333-44.

27. Itoh S, Hamada E, Kamoshida G, Yokoyama R, Takii T, Onozaki K, et al. Staphylococcal superantigenlike protein 10 (SSL10) binds to human immunoglobulin $\mathrm{G}(\mathrm{IgG})$ and inhibits complement activation via the classical pathway. Mol Immunol. 2010;47(4):932-8.

28. Patel D, Wines BD, Langley RJ, Fraser JD. Specificity of staphylococcal superantigen-like protein 10 toward the human IgG1 Fc domain. J Immunol. 2010;184(11):6283-92.

29. Itoh S, Yokoyama R, Kamoshida G, Fujiwara T, Okada H, Takii T, et al. Staphylococcal superantigenlike protein 10 (SSL10) inhibits blood coagulation by binding to prothrombin and factor Xa via their gamma-carboxyglutamic acid (Gla) domain. J Biol Chem. 2013;288(30):21569-80.

30. Itoh S, Yokoyama R, Murase C, Takii T, Tsuji T, Onozaki K. Staphylococcal superantigen-like protein 10 binds to phosphatidylserine and apoptotic cells. Microbiol Immunol. 2012;56(6):363-71.

31. Dutta D, Mukherjee D, Mukherjee IA, Maiti TK, Basak A, Das AK. Staphylococcal superantigen-like proteins interact with human MAP kinase signaling protein ERK2. FEBS Lett. 2019.

32. Wang H, Sun L, Su L, Rizo J, Liu L, Wang LF, et al. Mixed lineage kinase domain-like protein MLKL causes necrotic membrane disruption upon phosphorylation by RIP3. Mol Cell. 2014;54(1):133-46.

33. Laster SM, Wood JG, Gooding LR. Tumor necrosis factor can induce both apoptic and necrotic forms of cell lysis. Journal of immunology. 1988;141(8):2629-34.

34. Vercammen D, Brouckaert G, Denecker G, Van de Craen M, Declercq W, Fiers W, et al. Dual signaling of the Fas receptor: initiation of both apoptotic and necrotic cell death pathways. The Journal of experimental medicine. 1998;188(5):919-30.

35. Vanden Berghe T, Linkermann A, Jouan-Lanhouet S, Walczak H, Vandenabeele P. Regulated necrosis: the expanding network of non-apoptotic cell death pathways. Nat Rev Mol Cell Biol. 2014;15(2):13547.

36. Pasparakis $M$, Vandenabeele P. Necroptosis and its role in inflammation. Nature. 2015;517(7534):311-20.

37. Dai JL, Zhang CH, Guo L, He H, Jiang KY, Huang YX, et al. A necroptotic-independent function of MLKL in regulating endothelial cell adhesion molecule expression. Cell death \& disease. 2020;11(4).

38. He S, Wang L, Miao L, Wang T, Du F, Zhao L, et al. Receptor interacting protein kinase-3 determines cellular necrotic response to TNF-alpha. Cell. 2009;137(6):1100-11.

39. Hanggi K, Vasilikos L, Valls AF, Yerbes R, Knop J, Spilgies LM, et al. RIPK1/RIPK3 promotes vascular permeability to allow tumor cell extravasation independent of its necroptotic function. Cell death \& disease. 2017;8(2):e2588.

40. Strilic B, Yang L, Albarran-Juarez J, Wachsmuth L, Han K, Muller UC, et al. Tumour-cell-induced endothelial cell necroptosis via death receptor 6 promotes metastasis. Nature. 2016;536(7615):2158.

41. Ying Y, Padanilam BJ. Regulation of necrotic cell death: p53, PARP1 and cyclophilin D-overlapping pathways of regulated necrosis? Cell Mol Life Sci. 2016;73(11-12):2309-24. 
42. Arcus VL, Langley R, Proft T, Fraser JD, Baker EN. The Three-dimensional structure of a superantigenlike protein, SET3, from a pathogenicity island of the Staphylococcus aureus genome. J Biol Chem. 2002;277(35):32274-81.

43. Al-Shangiti AM, Naylor CE, Nair SP, Briggs DC, Henderson B, Chain BM. Structural relationships and cellular tropism of staphylococcal superantigen-like proteins. Infect Immun. 2004;72(7):4261-70.

44. Baker HM, Basu I, Chung MC, Caradoc-Davies T, Fraser JD, Baker EN. Crystal structures of the staphylococcal toxin SSL5 in complex with sialyl Lewis $X$ reveal a conserved binding site that shares common features with viral and bacterial sialic acid binding proteins. J Mol Biol. 2007;374(5):1298308.

45. Hermans SJ, Baker HM, Sequeira RP, Langley RJ, Baker EN, Fraser JD. Structural and functional properties of staphylococcal superantigen-like protein 4. Infect Immun. 2012;80(11):4004-13.

46. Koymans KJ, Feitsma LJ, Brondijk TH, Aerts PC, Lukkien E, LossI P, et al. Structural basis for inhibition of TLR2 by staphylococcal superantigen-like protein 3 (SSL3). Proc Natl Acad Sci U S A. 2015;112(35):11018-23.

47. Itoh S, Takii T, Onozaki K, Tsuji T, Hida S. Identification of the blood coagulation factor interacting sequences in staphylococcal superantigen-like protein 10. Biochem Biophys Res Commun. 2017;485(1):201-8.

48. Weiss SL, Fitzgerald JC, Pappachan J, Wheeler D, Jaramillo-Bustamante JC, Salloo A, et al. Global epidemiology of pediatric severe sepsis: the sepsis prevalence, outcomes, and therapies study. Am J Respir Crit Care Med. 2015;191(10):1147-57.

49. Schlapbach LJ, MacLaren G, Festa M, Alexander J, Erickson S, Beca J, et al. Prediction of pediatric sepsis mortality within $1 \mathrm{~h}$ of intensive care admission. Intensive Care Med. 2017;43(8):1085-96.

50. Lacey KA, Geoghegan JA, McLoughlin RM. The Role of Staphylococcus aureus Virulence Factors in Skin Infection and Their Potential as Vaccine Antigens. Pathogens. 2016;5(1).

51. Wilke GA, Bubeck Wardenburg J. Role of a disintegrin and metalloprotease 10 in Staphylococcus aureus alpha-hemolysin-mediated cellular injury. Proceedings of the National Academy of Sciences of the United States of America. 2010;107(30):13473-8.

52. Genestier AL, Michallet MC, Prevost G, Bellot G, Chalabreysse L, Peyrol S, et al. Staphylococcus aureus Panton-Valentine leukocidin directly targets mitochondria and induces Bax-independent apoptosis of human neutrophils. J Clin Invest. 2005;115(11):3117-27.

53. Goodyear CS, Silverman GJ. Death by a B cell superantigen: In vivo VH-targeted apoptotic supraclonal B cell deletion by a Staphylococcal Toxin. J Exp Med. 2003;197(9):1125-39.

54. den Reijer PM, Lemmens-den Toom N, Kant S, Snijders SV, Boelens H, Tavakol M, et al. Characterization of the humoral immune response during Staphylococcus aureus bacteremia and global gene expression by Staphylococcus aureus in human blood. PLoS One. 2013;8(1):e53391.

55. Draaijers L, Hassing RJ, Kooistra M, van Kessel K, Hovens M. Severe Acquired Coagulopathy During Fulminant Staphylococcus aureus Sepsis Most Likely Caused by S. aureus Exotoxins (SSLs). Eur J Case Rep Intern Med. 2018;5(12):0001002.

Page 20/30 
56. Wang F, Zhang N, Li B, Liu L, Ding L, Wang Y, et al. Heparin defends against the toxicity of circulating histones in sepsis. Front Biosci (Landmark Ed). 2015;20:1259-70.

57. Bae T, Schneewind O. Allelic replacement in Staphylococcus aureus with inducible counter-selection. Plasmid. 2006;55(1):58-63.

58. Otwinowski Z MW. Processing of X-ray diffraction data collected in oscillation mode. Methods Enzymol. 1997;276:307-26.

59. McCoy AJ, Grosse-Kunstleve RW, Adams PD, Winn MD, Storoni LC, Read RJ. Phaser crystallographic software. J Appl Crystallogr. 2007;40(Pt 4):658-74.

60. Collaborative. The CCP4 suite: programs for protein crystallography. Acta Crystallographica Section D. 1994;50(5):760-3.

61. Vagin AA, Steiner RA, Lebedev AA, Potterton L, McNicholas S, Long F, et al. REFMAC5 dictionary: organization of prior chemical knowledge and guidelines for its use. Acta Crystallogr D Biol Crystallogr. 2004;60(Pt 12 Pt 1):2184-95.

62. Adams PD, Afonine PV, Bunkoczi G, Chen VB, Davis IW, Echols N, et al. PHENIX: a comprehensive Python-based system for macromolecular structure solution. Acta Crystallographica Section D. 2010;66(2):213-21.

63. Emsley P, Lohkamp B, Scott WG, Cowtan K. Features and development of Coot. Acta Crystallographica Section D. 2010;66(4):486-501.

\section{Figures}


A

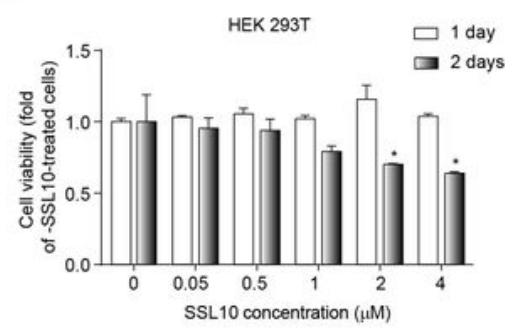

B

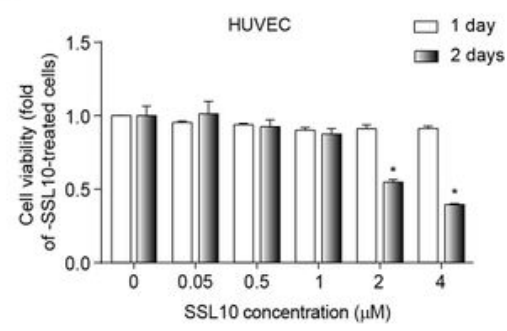

E

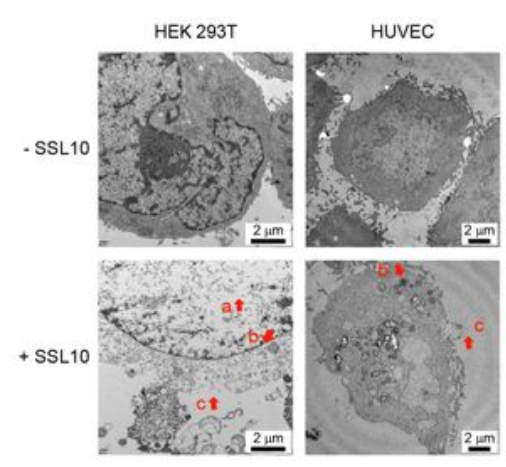

G

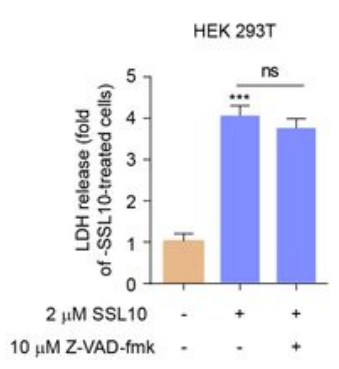

C
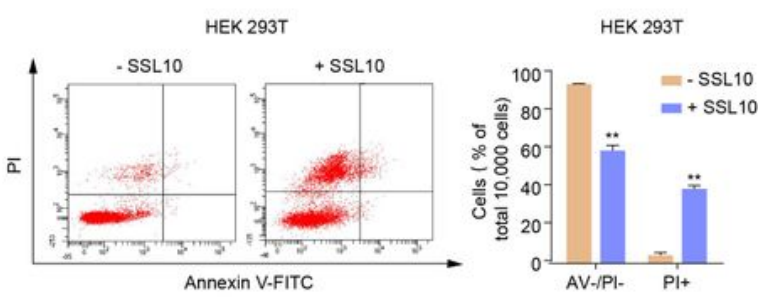

D

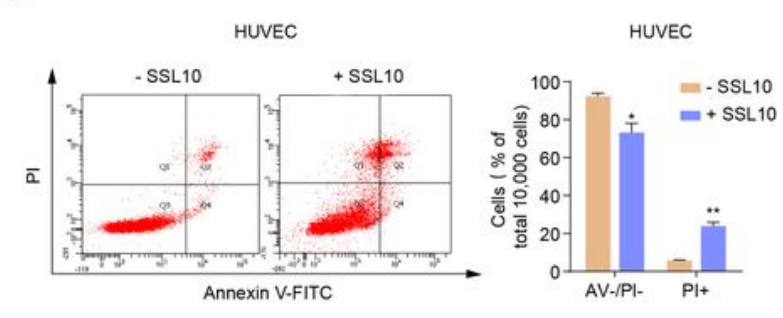

F
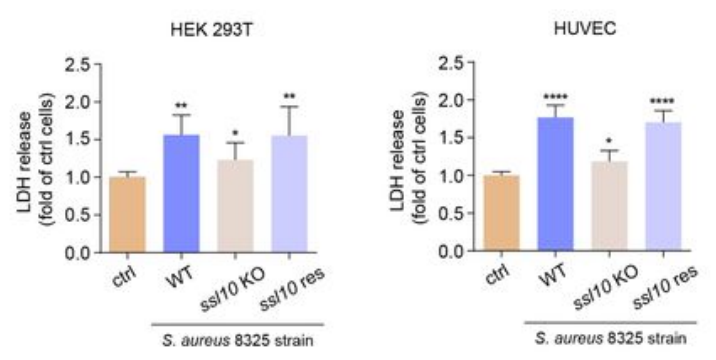

H

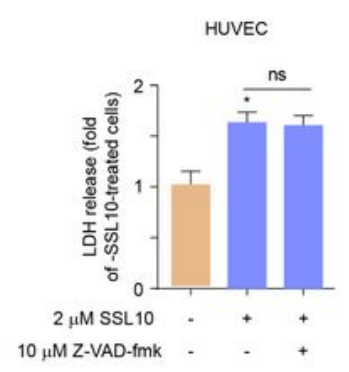

\section{Figure 1}

SSL10 induces necrosis in HEK 293T and HUVEC cells. (A, B) HEK 293T (A) and HUVEC cells (B) were treated with different concentrations of SSL10 for different time periods, as indicated. SSL10 induces cell death in a dose- and time-dependent manner as determined by MTS assay. (C, D) HEK 293T (C) and HUVEC cells (D) were collected after treatment with $2 \mu \mathrm{M}$ SSL10 for $48 \mathrm{~h}$. Compromised cells were then detected by flow cytometry using Annexin V/PI staining. The dot plot (left) is representative of three 
independent experiments, and the quantification results are shown as a bar graph (right). (E) Transmission electron microscopy images of HEK 293T and HUVEC cells treated with or without $2 \mu \mathrm{M}$ SSL10 for $48 \mathrm{~h}$. Letters with arrows indicate characteristic features of necrotic morphology: a, cytoplasmic lightening; $b$, swollen organelle; $c$, membrane rupture. (F) The same amount of wild type $S$. aureus 8325 , or ss 110 knock out and ss 10 complementation bacteria in the $\mathrm{S}$. aureus 8325 background were cultured to the same cell density, and the supernatants were collected and used to treat HEK 293T and HUVEC cells for $48 \mathrm{~h}$ at 1:1000 dilution. LDH released from the cells was evaluated. WT, wild type. (G, H) The release of LDH into the culture medium of HEK 293T (G) or HUVEC cells (H) was significantly higher after treatment with SSL10 for $48 \mathrm{~h}$, indicated by increased absorbance at $490 \mathrm{~nm}$. The pancaspase inhibitor (Z-VAD-fmk, $10 \mu \mathrm{M}$ ) was applied 30 min before $2 \mu \mathrm{M} \mathrm{SSL} 10$ treatment, and did not abolish SSL10 cytotoxicity, as evident from LDH release. All data represent means \pm SD calculated from at least three independent experiments. ${ }^{*}, p<0.05 ; * \star, p<0.01 ; * \star *, p<0.001 ; * \star \star *, p<0.0001$ compared to those without SSL10 treatment (-SSL10-treated cells, i.e., buffer-treated cells) or ctrl cells (TSB mediumtreated cells) under the same conditions. ns, not significant.

A

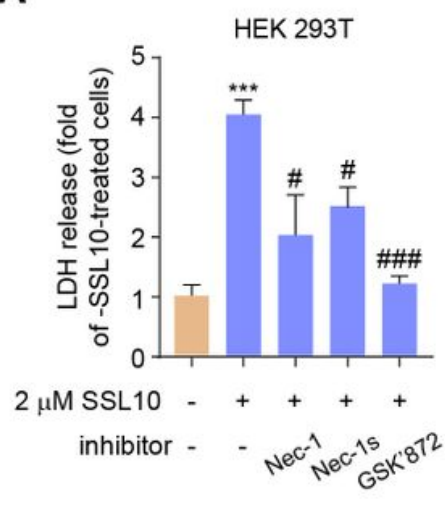

B

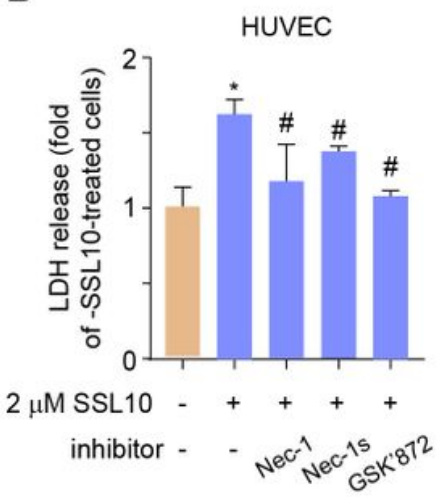

C

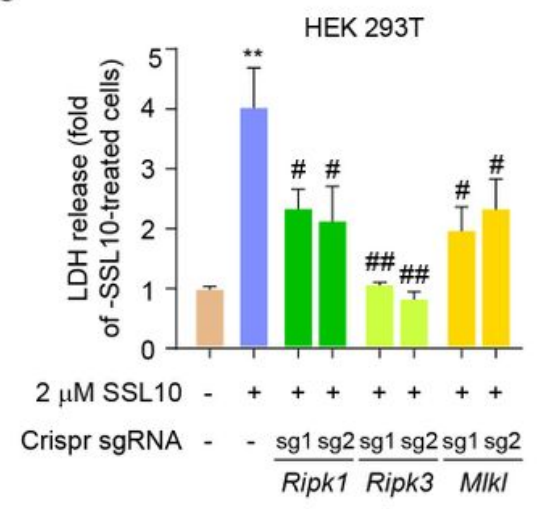

D

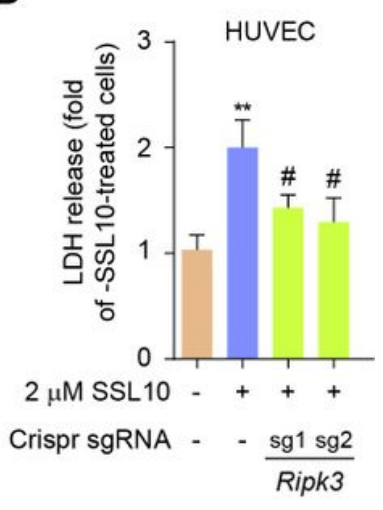

$\mathbf{E}$

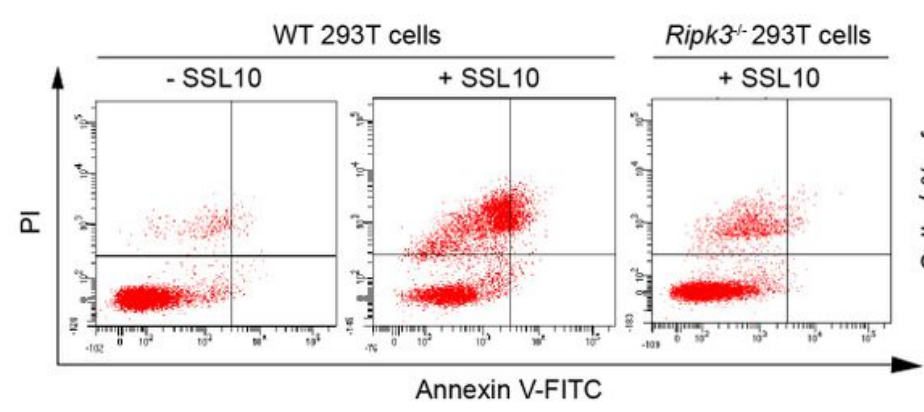

$\mathbf{F}$

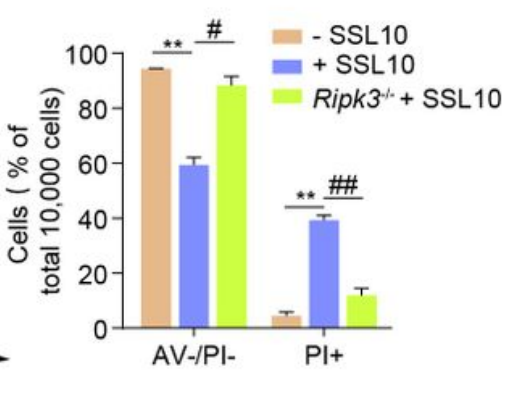

HEK 293 T

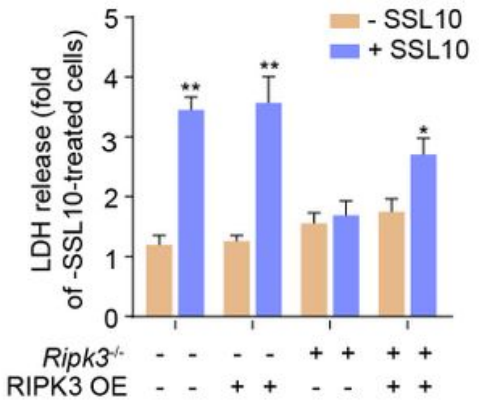

Figure 2

SSL10-induced necroptosis is RIPK3 dependent. (A, B) HEK 293T (A) and HUVEC cells (B) were treated with various inhibitors 30 min prior to treatment with $2 \mu \mathrm{M} \mathrm{SSL10}$. The release of LDH associated with 
SSL10 exposure could be inhibited by RIPK3 inhibitor GSK'872 $(50 \mu \mathrm{M})$, but was only partially inhibited by the RIPK1 inhibitors Nec-1 $(100 \mu \mathrm{M})$ or Nec-1s (50 $\mu \mathrm{M})$. (C) Ripk1, Ripk3, or Mlkl were knocked out with two different sgRNAs in HEK 293T cells. RIPK3-KO, but not RIPK1-KO or MLKL-KO, could abolish SSL10 cytotoxicity, evident from LDH release into the cell culture supernatant. (D) Ripk3 was knocked out with two different sgRNAs in HUVEC cells. RIPK3-KO could abolish SSL10 cytotoxicity to HEK 293T cells, evident from LDH release into the cell culture supernatant. (E) The absence of RIPK3 increased the viability of SSL10-treated HEK 293T cells, indicated by flow cytometry analysis with Annexin V/PI staining. The dot plot (left) is representative of three independent experiments, and the results of quantification (right) are shown as a bar graph. (F) Transient complementation of RIPK3 in RIPK3-KO HEK 293T cells resulted in a significant increase in LDH release. All data represent means \pm SD calculated from at least three independent experiments. *, $p<0.05 ; * \star, p<0.01 ; * \star *, p<0.001$ compared to those without SSL10 treatment (-SSL10-treated cells, i.e., buffer- treated cells) under the same conditions. \#, $p<$ $0.05 ; \# \#, p<0.01 ; \# \# \#, p<0.001$ compared to those treated with $2 \mu \mathrm{M}$ SSL10 treatment for $48 \mathrm{~h}$ under the same conditions. 
A

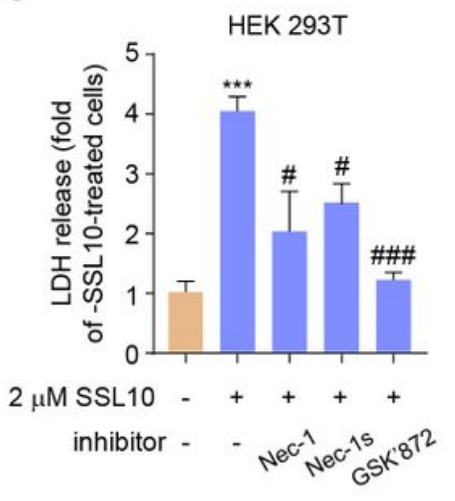

E

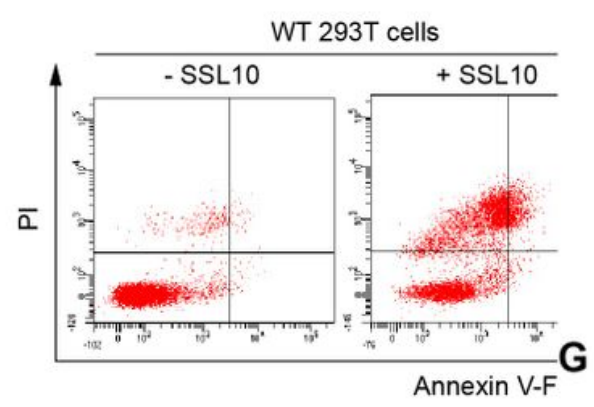

B A

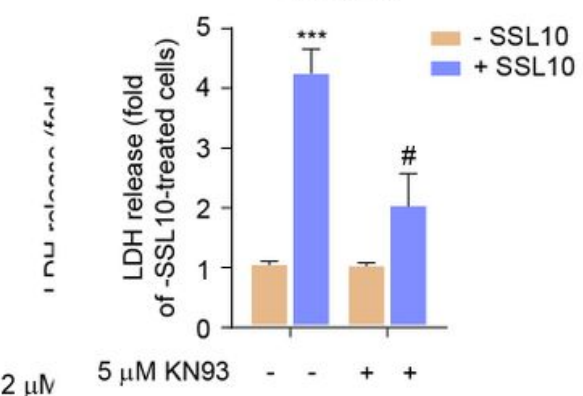

D

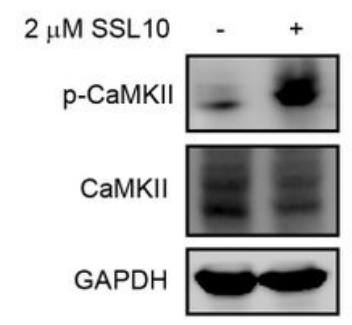

B

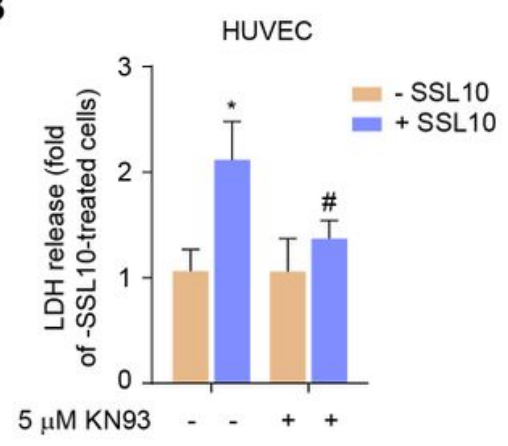

E

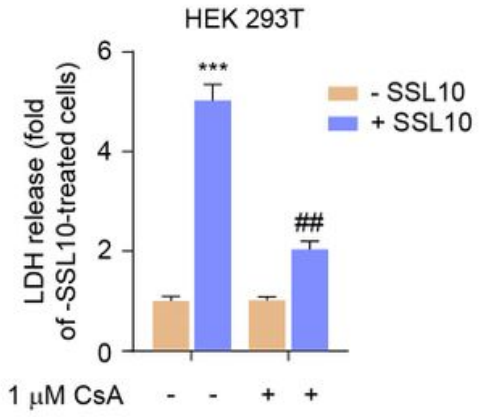

C

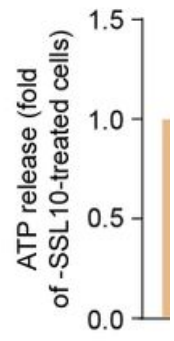

$5 \mu \mathrm{M} \mathrm{KN93}$

\section{$\mathbf{F}$}

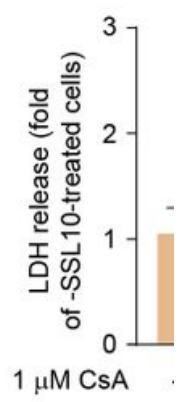

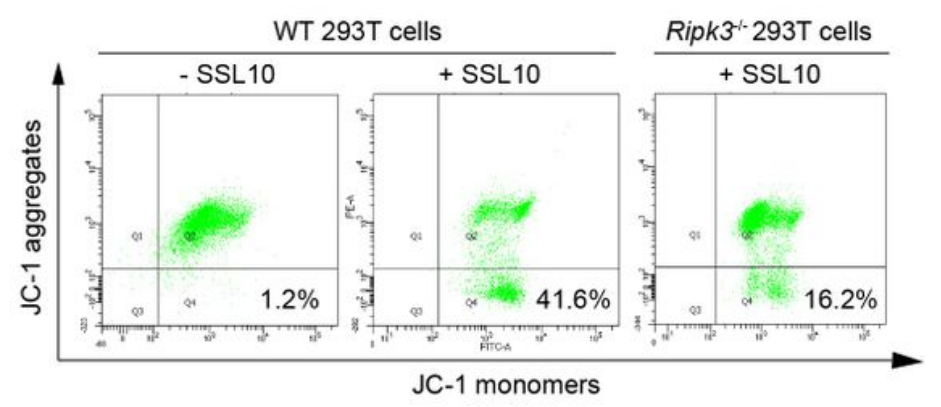

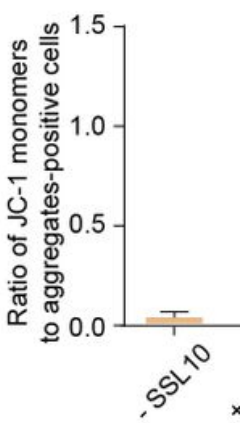

\section{Figure 3}

CaMKII activation and mPTP opening are essential for SSL10-induced necroptosis in HEK 293T and HUVEC cells. (A, B) HEK 293T (A) or HUVEC cells (B) were pretreated with CaMKII inhibitor KN-93 (5 $\mu$ M) 30 min before SSL10 treatment, and release of LDH to the medium was determined. (C) ATP release after HEK 293T cells were treated with SSL10 with or without KN-93 pretreatment. (D) CaMKII and phosphoThr287 CaMKII were detected by Western blotting in whole cell lysates of HEK 293T cells treated with or without SSL10. The graph is representative of three independent experiments. (E, F) HEK 293T (E) and HUVEC cells $(F)$ were pretreated with MPTP inhibitor Cyclosporin A (CsA, $1 \mu \mathrm{M}) 30$ min before SSL10 treatment and LDH release into the culture media was quantified. (G) Depolarization of the mitochondrial membrane of HEK 293T cells induced by SSL10 was reduced in the absence of RIPK3, as measured by flow cytometry after JC-1 staining. The dot plot (left) is representative of three independent experiments, 
and the results of quantification are shown as a bar graph (right). All data represent the means $\pm S D$ calculated from at least three independent experiments. ${ }^{*}, p<0.05 ;{ }^{* *}, p<0.01 ; * \star *, p<0.001$ compared to those without SSL10 treatment (-SSL10-treated cells, i.e., buffer-treated cells) under the same conditions. $\#, \mathrm{p}<0.05 ; \# \#, \mathrm{p}<0.01$ compared to those treated with $2 \mu \mathrm{M} S \mathrm{SS} 10$ for $48 \mathrm{~h}$ under the same conditions.

A

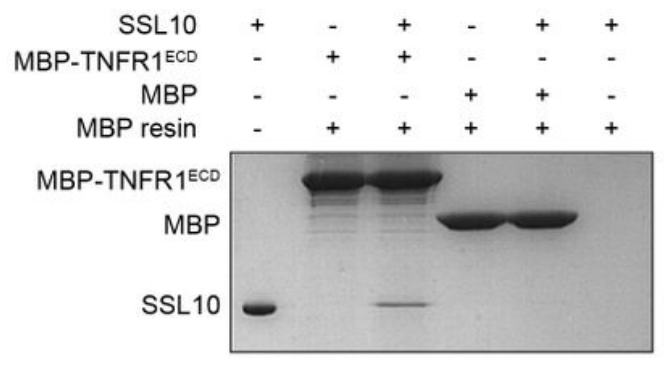

B

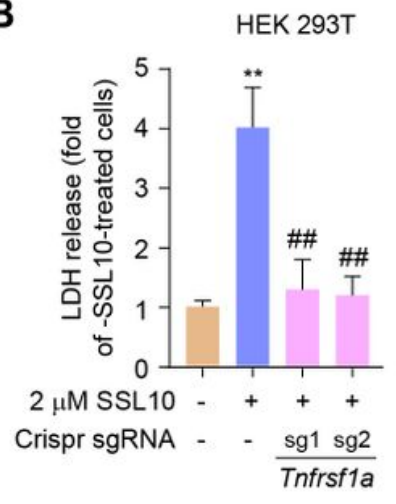

C

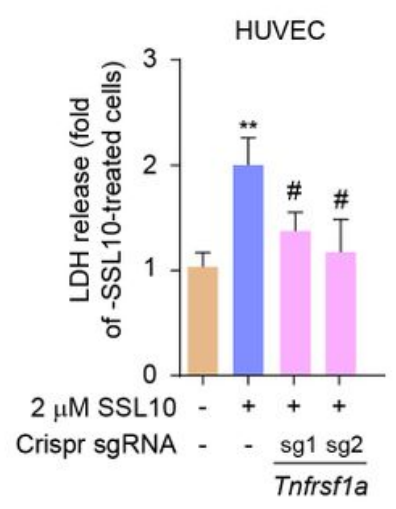

D
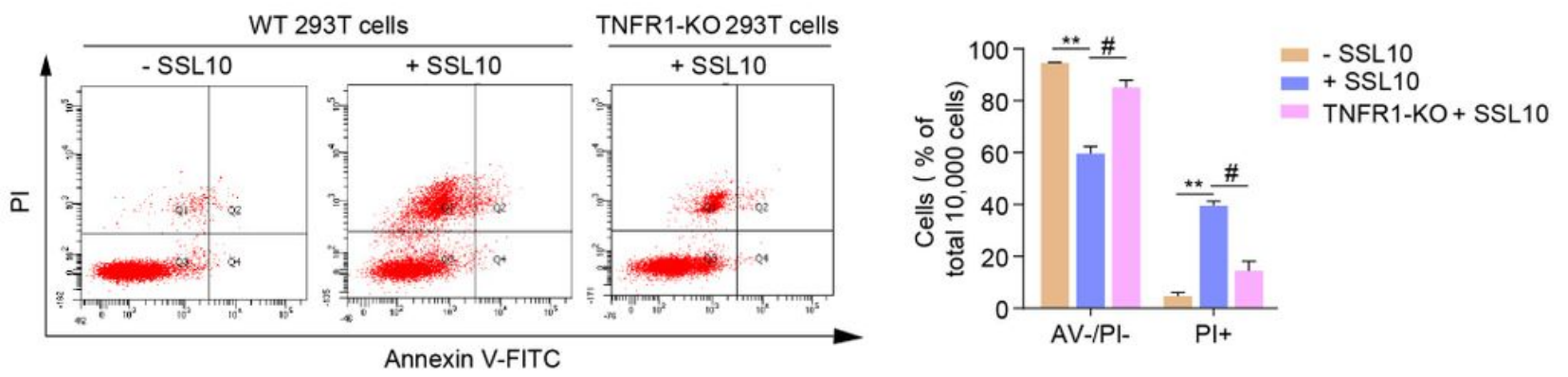

E
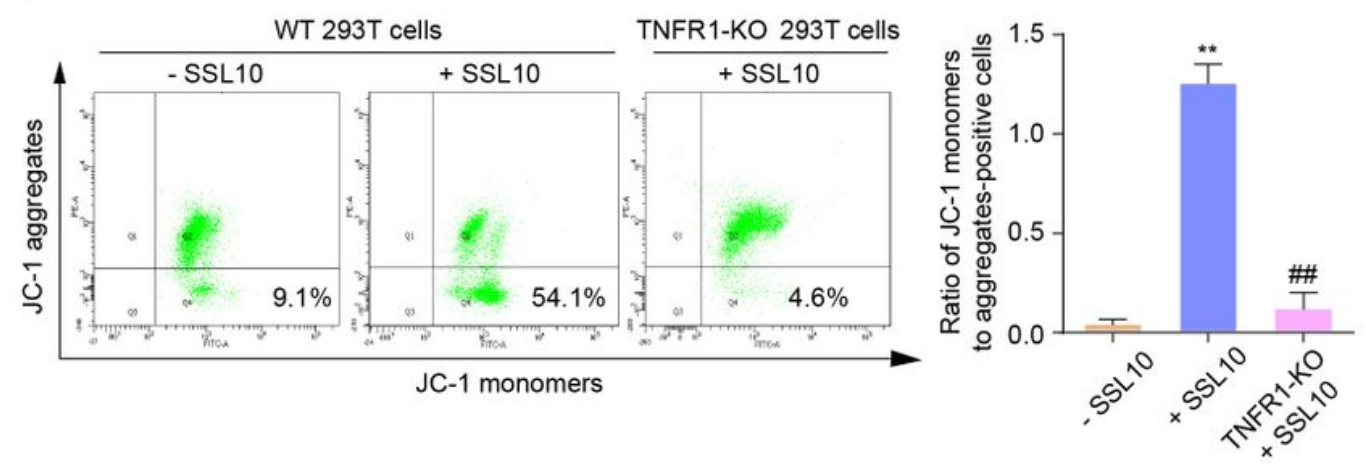

F

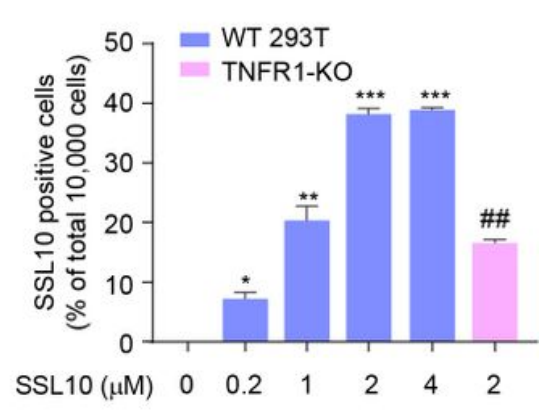

\section{Figure 4}

SSL10 induces necroptosis by direct interaction with the extracellular domain of TNFR1 (TNFR1ECD). (A) MBP pull-down of SSL10 by MBP-TNFR1ECD. (B, C) Knockout of TNFR1 abolished SSL10-induced cytotoxicity in HEK 293T (B) or HUVEC cells (C), indicated by diminished release of LDH. (D) The absence of TNFR1 increased viability of SSL10-treated HEK 293T cells, as measured by flow cytometry after 
Annexin V/PI staining. (E) Depolarization of the mitochondrial membrane of HEK 293T cells induced by SSL10 was reduced in the absence of TNFR1, measured by flow cytometry after JC-1 staining. The dot plot (left) is representative of three independent experiments, and the results of quantification (right) are shown as a bar graph. (F) SSL10 at different concentrations was incubated with WT or TNFR1-KO HEK 293 T cells for $30 \mathrm{~min}$, and SSL10 bound to the cell surface was detected by flow cytometry using FITCconjugated anti-His-tag antibody binding. The absence of TNFR1 significantly reduced binding of SSL10 to the cell surface. All data represent means \pm SD calculated from at least three independent experiments. $\star, p<0.05 ; * \star, p<0.01 ; * \star \star, p<0.001$ compared to those without SSL10 treatment (-SSL10-treated cells, i.e., buffer-treated cells) under the same conditions. \#, $p<0.05$; \#\#, $p<0.01$ compared to those treated with $2 \mu \mathrm{M}$ SSL10 for $48 \mathrm{~h}$ under the same conditions.

A

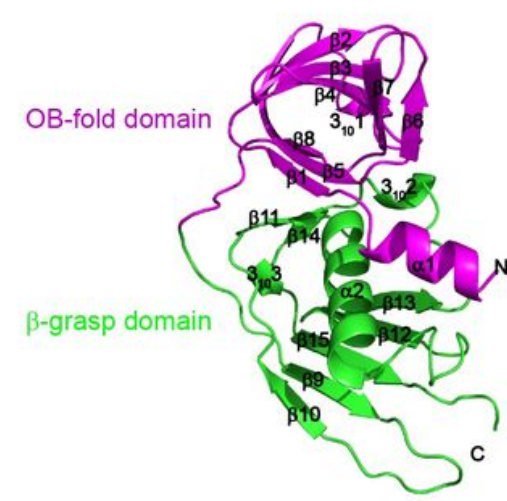

C

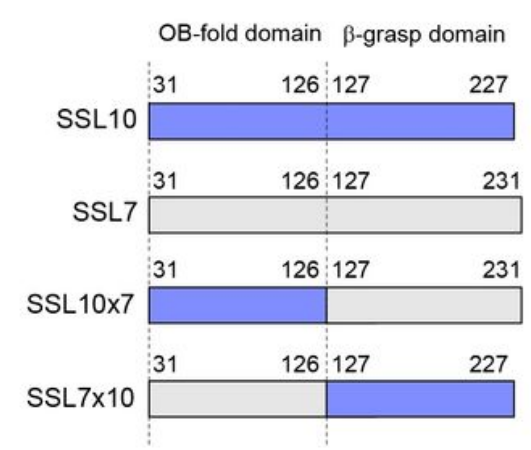

B

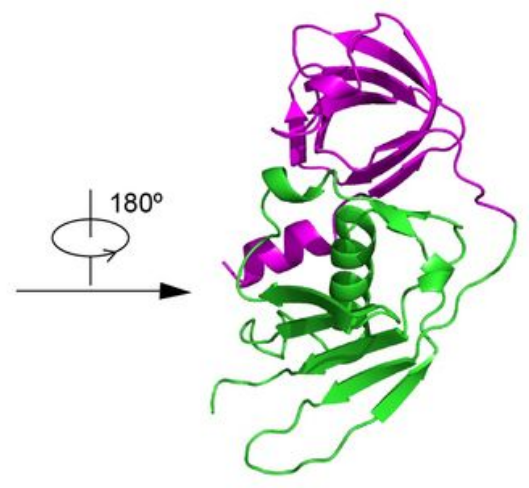

D

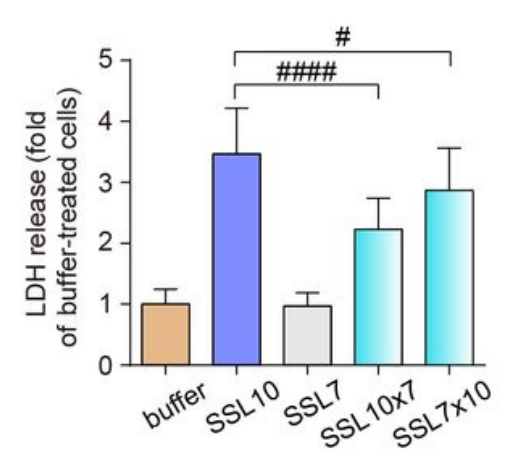

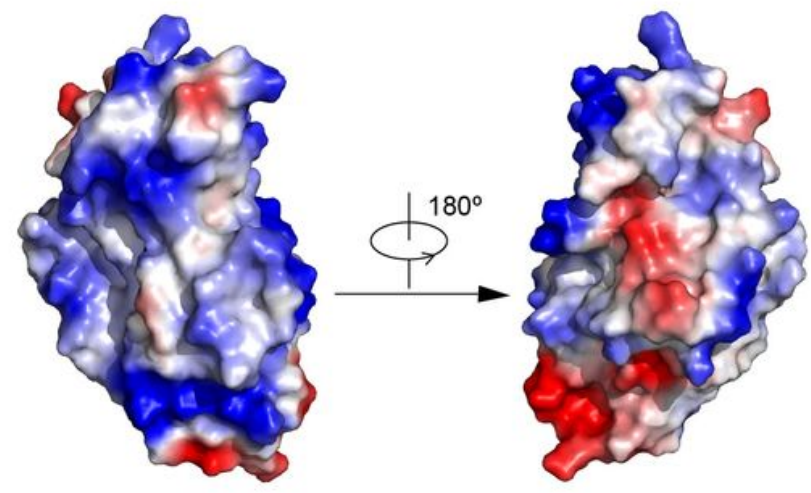

$\mathbf{E}$

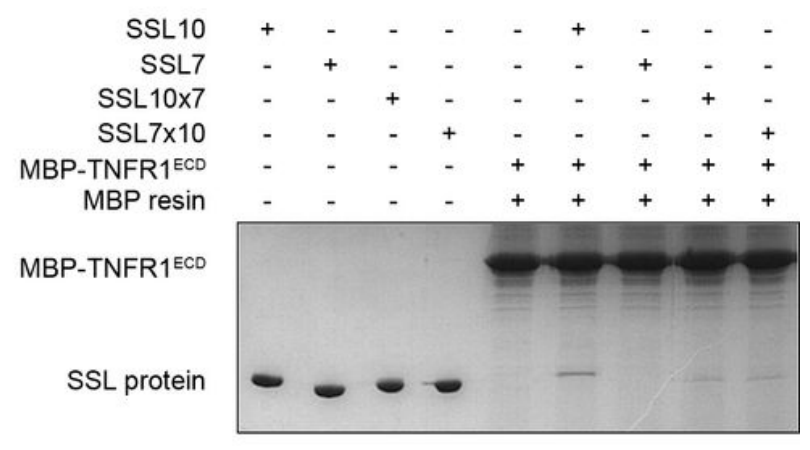

\section{Figure 5}

Both the $\mathrm{N}$ - and $\mathrm{C}$ - terminal domains contribute to SSL10-induced necroptosis. (A) The overall structure of SSL10. The OB-fold domain and $\beta$-grasp domain of SSL10 are colored magenta and green, respectively. (B) The electrostatic surface potentials of SSL10. Positively and negatively charged surface regions are colored blue and red, respectively. (C) Illustration of chimeric SSL variants used in this study. Blue and 
gray boxes represent sequences derived from SSL10 and SSL7, respectively. The numbers indicate the residue positions of the $\mathrm{N}$ - and C-terminus of the swapped domains. (D) LDH released from HEK 293T cells treated with $2 \mu \mathrm{M}$ SSL10, SSL7, SSL10'7, and SSL7'10. All data represent means \pm SD calculated from at least three independent experiments. \#, $p<0.05$; \#\#\#, $p<0.0001$ as indicated. (E) MBP pulldown of SSL10, SSL7, SSL10'7 and SSL7'10 by MBP-TNFR1ECD.

A

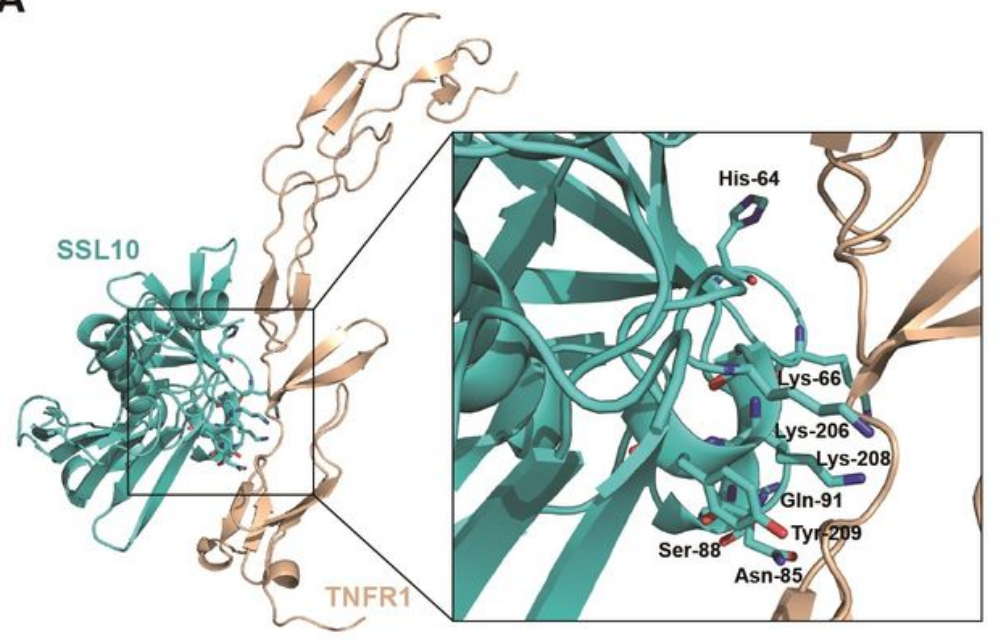

C

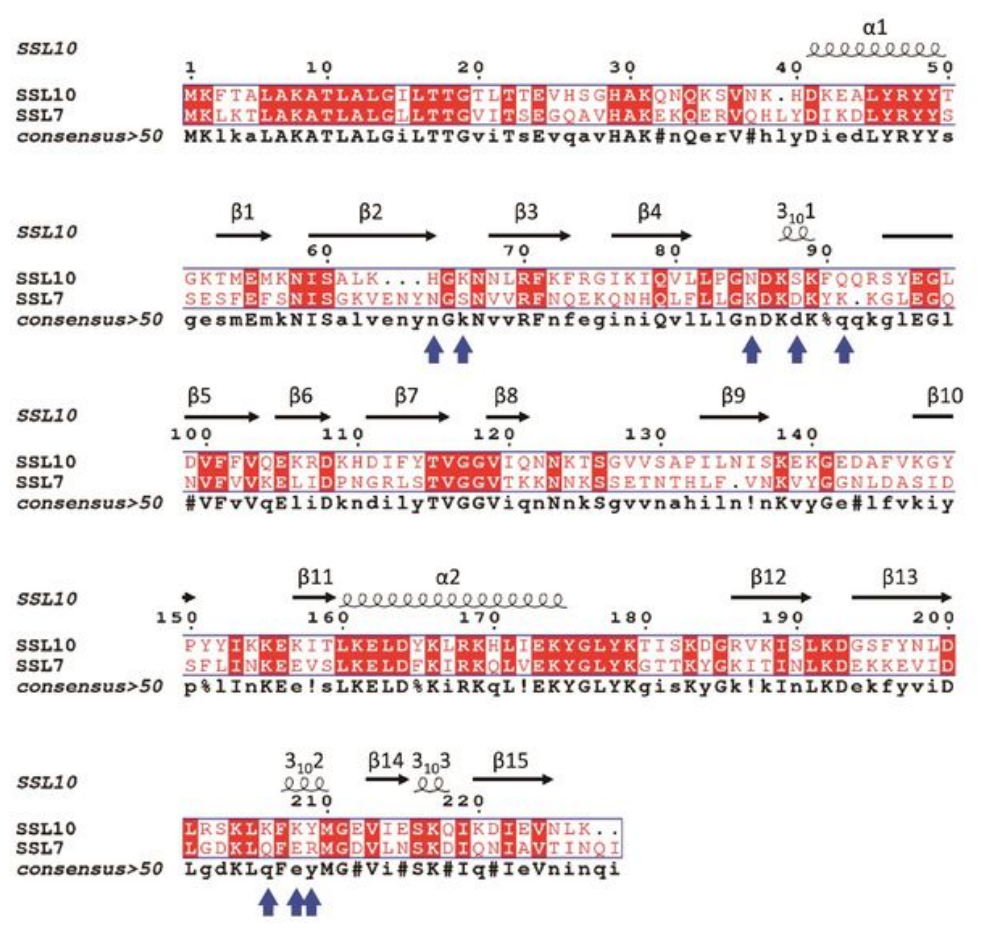

B

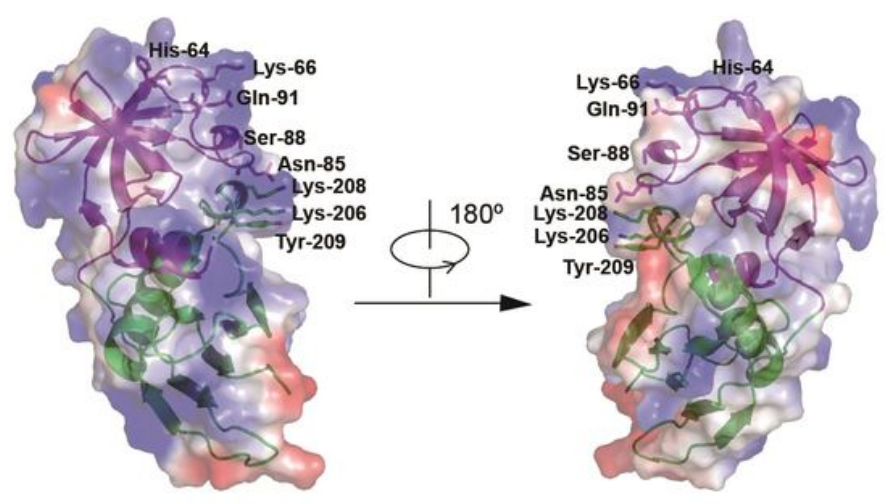

D

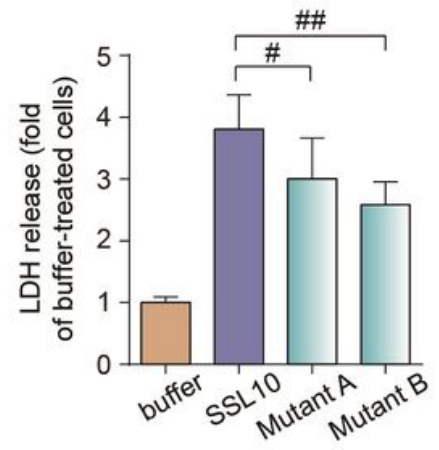

$\mathbf{F}$

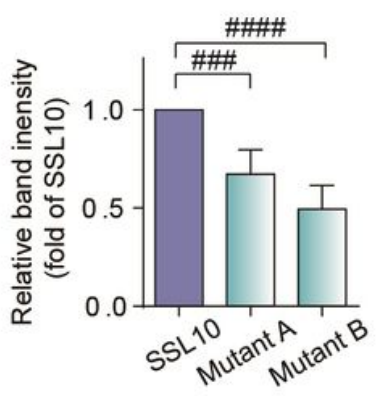

E

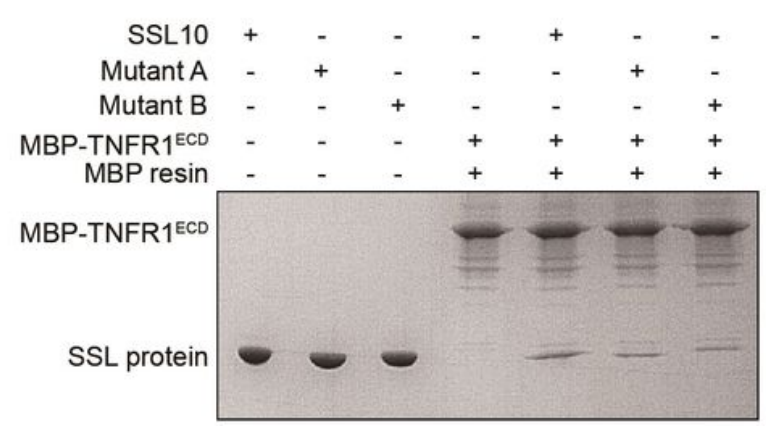

Figure 6

Identification of potential TNFR1 binding sites on SSL10. (A) The structures of SSL10 and TNFR1ECD (PDB code: 1EXT) were docked using the HDOCK program to generate structural models of the 
SSL10/TNFR1ECD complex. Enlarged view shows the residues of SSL10 possibly involved in binding to TNFR1ECD. SSL10 and TNFR1ECD are colored cyan and light brown, respectively. Amino acid residues are shown as sticks. (B) The potential TNFR1 binding sites on SSL10. The electrostatic surface potentials of SSL10 are shown. Positively and negatively charged surface regions are colored blue and red, respectively. (C) Sequence alignment of SSL10 and SSL7 was conducted with MultAlin and ESPript. Amino acid residues of SSL10 possibly involved in the interaction with the TNFR1ECD are indicated by blue arrows. (D) LDH released from HEK 293T cells treated with $2 \mu \mathrm{M} \mathrm{SSL10}$ or either of the two mutants. The eight residues potentially involved in SSL10-TNFR1 interface, i.e., H64, K66, N85, S88, Q91, K206, K208, and Y209 in SSL10, were replaced with either alanine residues (mutant A) or with the corresponding residues from SSL7 (mutant B). (E) MBP pull-down of SSL10 and two mutants by MBPTNFR1ECD. (F) Quantification of the band intensity in (E). The relative band intensities of wild type or the two mutant SSL10 proteins pulled down (lane 5-7) are quantitated by densitometry after normalization to their input (lane 1-3), and then expressed as the fold of wild type SSL10. All data represent means \pm SD

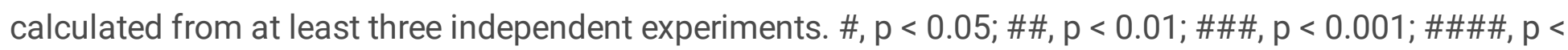
0.0001 as indicated. 


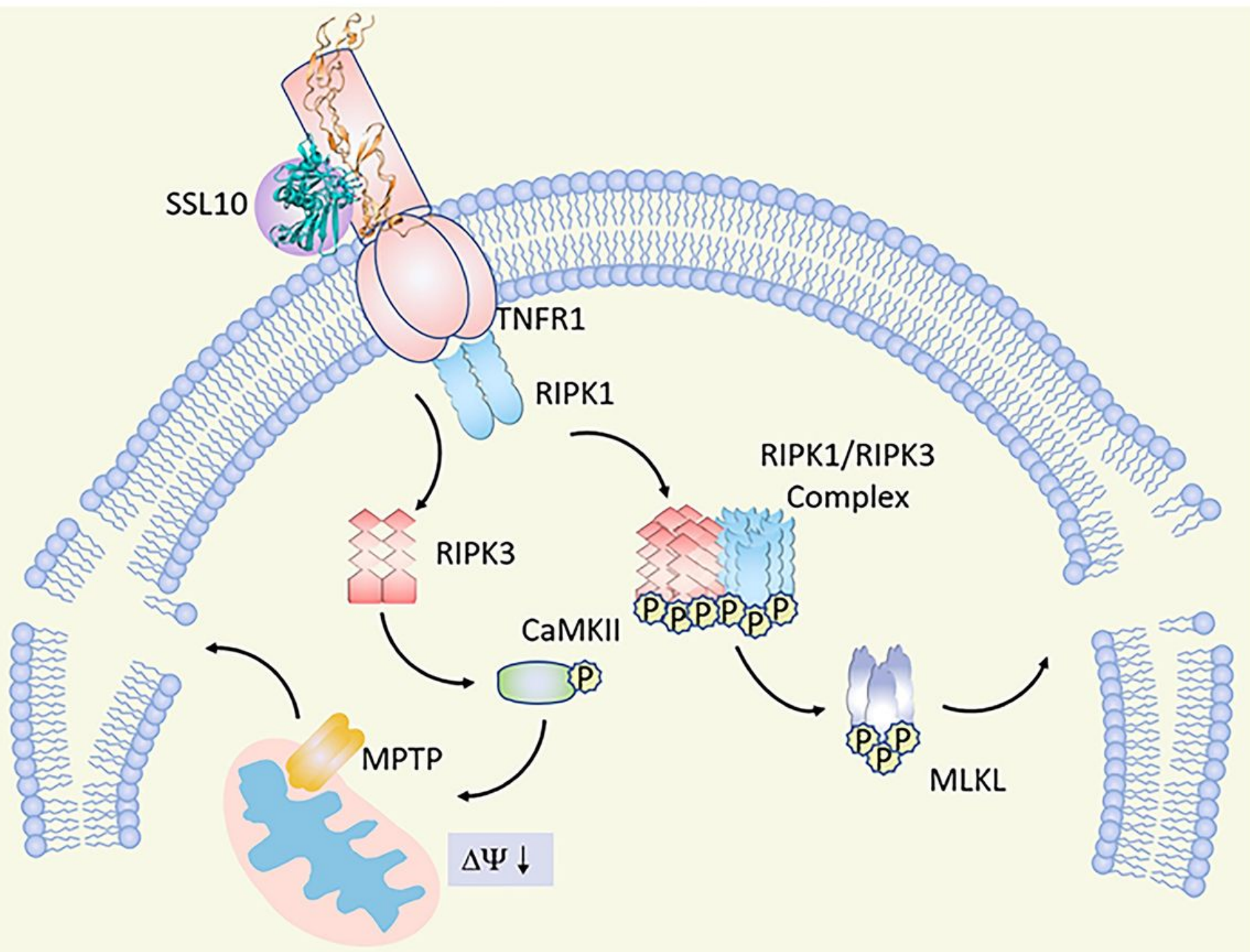

\section{Figure 7}

Schematic diagram of the SSL10 signal cascade and subsequent cellular effects. SSL10 secreted by S. aureus directly interacts with TNFR1 on the host cell surface and induces cell necroptosis via two distinct pathways, including the RIPK1-RIPK3-MLKL and RIPK3-CaMKII-mPTP pathways.

\section{Supplementary Files}

This is a list of supplementary files associated with this preprint. Click to download.

- SupplementaryInformation.docx 